\title{
A muscle-specific UBE20/AMPKa2 axis promotes insulin resistance and metabolic syndrome in obesity
}

\author{
Isabelle K. Vila, ${ }^{1}$ Mi Kyung Park, ${ }^{1}$ Stephanie Rebecca Setijono, ${ }^{2}$ Yixin Yao, ${ }^{1}$ Hyejin Kim, ${ }^{1}$ \\ Pierre-Marie Badin, ${ }^{3}$ Sekyu Choi, ${ }^{4}$ Vihang Narkar, ${ }^{3}$ Sung-Woo Choi, ${ }^{5}$ Jongkyeong Chung, ${ }^{4}$ \\ Cedric Moro, ${ }^{6}$ Su Jung Song, ${ }^{2}$ and Min Sup Song ${ }^{1,7}$ \\ 'Department of Molecular and Cellular Oncology, The University of Texas MD Anderson Cancer Center, Houston, Texas, \\ USA. ${ }^{2}$ Soonchunhyang Institute of Medi-Bio Science, Soonchunhyang University, Cheonan-si, South Korea. ${ }^{3}$ Center for \\ Metabolic and Degenerative Diseases, Institute of Molecular Medicine, The University of Texas McGovern Medical School, \\ Houston, Texas, USA. ${ }^{4}$ School of Biological Sciences, Seoul National University, Seoul, South Korea. ${ }^{5}$ Department of \\ Orthopedic Surgery, Soonchunhyang University College of Medicine, Seoul, South Korea. ${ }^{6}$ Obesity Research Laboratory, \\ Institute of Metabolic and Cardiovascular Diseases, UMR 1048, Inserm, Toulouse, France. `CGraduate School of Biomedical \\ Sciences, The University of Texas MD Anderson Cancer Center, Houston, Texas, USA
}

Ubiquitin-conjugating enzyme E2O (UBE20) is expressed preferentially in metabolic tissues, but its role in regulating energy homeostasis has yet to be defined. Here we find that UBE2O is markedly upregulated in obese subjects with type 2 diabetes and show that whole-body disruption of Ube2o in mouse models in vivo results in improved metabolic profiles and resistance to high-fat dietinduced (HFD-induced) obesity and metabolic syndrome. With no difference in nutrient intake, $\mathrm{Ube}^{2 \mathrm{o}^{-/-}}$mice were leaner and expended more energy than WT mice. In addition, hyperinsulinemiceuglycemic clamp studies revealed that $U b e 20^{-/-}$mice were profoundly insulin sensitive. Through phenotype analysis of HFD mice with muscle-, fat-, or liver-specific knockout of Ube2o, we further identified UBE2O as an essential regulator of glucose and lipid metabolism programs in skeletal muscle, but not in adipose or liver tissue. Mechanistically, UBE2O acted as a ubiquitin ligase and targeted AMPK $\alpha 2$ for ubiquitin-dependent degradation in skeletal muscle; further, muscle-specific heterozygous knockout of Prkaa2 ablated UBE2O-controlled metabolic processes. These results identify the UBE20/AMPK $\alpha 2$ axis as both a potent regulator of metabolic homeostasis in skeletal muscle and a therapeutic target in the treatment of diabetes and metabolic disorders.

Authorship note: IKV and MKP contributed equally to this work.

Conflict of interest: The authors have declared that no conflict of interest exists.

Copyright: (c) 2019, American Society for Clinical Investigation.

Submitted: February 19, 2019

Accepted: May 24, 2019

Published: July 11, 2019.

Reference information: /CI Insight. 2019;4(13):e128269. https://doi. org/10.1172/ji.insight.128269

\section{Introduction}

Type 2 diabetes and metabolic syndrome, which constitute a serious health crisis worldwide, are associated with increased risks for nonalcoholic fatty liver disease (NAFLD) and cardiovascular disease and demand urgent therapeutic attention, especially for those at a high risk for these complications $(1,2)$. These diseases have been linked to the increased rates of obesity associated with overnutrition and sedentary lifestyle (1), and given the rising prevalence of obesity (3), identifying new insights into the molecular and genetic basis of diet-induced metabolic disturbance is of critical clinical importance. Insulin resistance is a fundamental pathogenic factor present in obesity and type 2 diabetes, and results in deregulation of glucose and lipid metabolism in muscle, fat, and liver tissue $(4,5)$. Although skeletal muscle primarily accounts for insulin-stimulated glucose disposal $(6,7)$, the mechanism underlying insulin resistance in muscle is poorly understood.

AMPK is a key sensor of cellular energy and nutrient levels. Once activated by falling energy status, AMPK activates ATP production by promoting glucose uptake and lipid oxidation through TBC1 domain family member 1 (TBC1D1) and acetyl-CoA carboxylase (ACC), respectively, and conserves ATP by switching off the processes of lipid, cholesterol, and protein synthesis (8). AMPK is also crucial in the regulation of metabolism and energy balance at the whole-body level (9). Increasing evidence indicates that AMPK plays important roles in the action mechanisms of metformin and rosiglitazone, as well as the biochemistry of exercise, which together represent the cornerstones of the clinical management of type 2 diabetes and metabolic syndrome (10-12). 
Research has therefore increasingly focused on identifying AMPK regulation mechanisms as new therapeutic targets for metabolic diseases. AMPK is a heterotrimeric serine/threonine kinase composed of catalytic $\alpha$ and regulatory $\beta$ and $\gamma$ subunits (8). In addition to the regulation of its enzymatic activity by adenine nucleotides and upstream kinases (such as LKB1/STK11 and CaMKK $\beta$ ) (8), accumulating evidence has indicated that the degradation of AMPK is precisely regulated (13-16). However, the molecular and physiological mechanisms underlying the selective modulation of AMPK subunit isoforms in the development of metabolic disease are so far unclear.

Ubiquitination requires the coordinated action of E1 (activating), E2 (conjugating), and E3 (ligating) enzymes (17). There are approximately $30 \mathrm{E} 2 \mathrm{~s}$ in the human genome, and while many are thought to be directly involved in ubiquitin transfer, very little functional information is available for the majority of them (18). The ubiquitin-conjugating E2O (UBE2O) is a relatively large E2 and functions as an E2/E3 hybrid, displaying both E2 and E3 activities (19-21). UBE2O has been implicated in multiple cellular processes, including adipogenesis, erythroid differentiation, protein trafficking, and interleukin signaling (21-27).

Through the characterization of Ube2o-knockout mice crossed with mouse cancer models, we recently demonstrated an important role for $\mathrm{UBE} 2 \mathrm{O}$ as an oncogene promoting tumor initiation, growth, and metastasis (16). Intriguingly, noncancerous tissue expression of UBE2O occurs preferentially in metabolic tissues such as the brain, skeletal muscle, adipose tissue, and liver (28), and yet critical questions about its tissue-specific roles in the regulation of metabolic homeostasis remain to be answered. To precisely define in vivo the physiological roles of UBE2O in energy homeostasis, we generated mice lacking Ube2o in the skeletal muscle, adipose tissue, or liver. Our subsequent findings showed that loss of Ube2o in skeletal muscle, but not in adipose or liver tissue, leads to improved glucose and lipid metabolism and protects mice from diet-induced insulin resistance and metabolic disorders

\section{Results}

UBE2O expression is markedly elevated in obese subjects with type 2 diabetes. To identify candidate ubiquitin-conjugating enzymes or E2s that regulate glucose homeostasis, we screened a synthetic siRNA library (4 siRNAs per gene) targeting 30 human and mouse E2s (18) in human and mouse skeletal muscle myotubes, and examined their respective rates of insulin-stimulated glucose uptake. After target deconvolution of observed hits, UBE2O was identified as a potent E2 negatively regulating glucose transport (Figure 1A). As obesity caused by excess feeding (or overnutrition) has become a problem of epidemic proportions, and is an underlying cause of metabolic disorders including type 2 diabetes and metabolic syndrome ( 1 , 3 ), we assessed the expression profiles of $E 2$ genes in mice fed a high-fat diet (HFD; $45 \%$ fat, $24 \%$ protein, and $41 \%$ carbohydrates, $4.7 \mathrm{kcal} / \mathrm{g}$ ) by comparative quantitative reverse transcription PCR (RT-qPCR). Notably, skeletal muscle Ube2o was highly expressed in HFD mice compared with mice fed normal chow (Figure 1B). Likewise, the levels of UBE2O protein rose strikingly in the skeletal muscle of HFD-induced obese mice or genetically diabetic $d b / d b$ mice compared with mice on chow or age-matched $d b /+$ lean mice (Figure 1, C and D). These results provide a critical clue to the link between UBE2O and metabolic disease.

Whole-body knockout of Ube2o protects mice against diet-induced metabolic syndrome. To determine whether UBE2O is required for the pathogenesis of metabolic syndrome, we used Ube2o-deficient $\left(U b e 20^{-/-}\right)$ mice and their WT littermates (Supplemental Figure 1A; supplemental material available online with this article; https://doi.org/10.1172/jci.insight.128269DS1) to track changes in body weight and metabolic parameters in response to HFD or chow. When challenged with HFD for 21 weeks, Ube $20^{-1-}$ mice gained significantly less body weight relative to WT counterparts (Figure 2A and Supplemental Figure

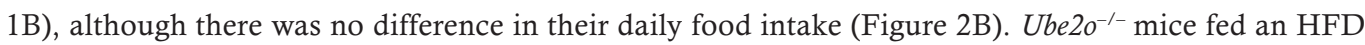
also exhibited a marked reduction in overall fat mass (Figure $2 \mathrm{C}$ and Supplemental Figure 1C), as visceral and abdominal subcutaneous fat masses were both markedly reduced (Figure 2D). Sections of white adipose tissue (WAT) from $U b e 20^{-1-}$ mice on an HFD also showed a marked reduction in adipocyte size (Figure 2E). Moreover, plasma lipid profiles revealed reductions in total cholesterol (TC), free fatty acid (FFA), and LDL levels (indicating dyslipidemia) (29) in Ube2 $\mathrm{o}^{-1-}$ mice on an HFD, whereas HDL levels remained stable (Figure $2 \mathrm{~F}$ ). Obesity often induces abnormal accumulation of triglycerides in the liver (hepatic steatosis or fatty infiltration of liver - the hallmark of NAFLD) (30), which can further deteriorate fat metabolism. We therefore examined the livers of $U b e 2 o^{-1-}$ and control mice fed an HFD. Liver weight and hepatic triglyceride measurement as well as histological analysis of $U b e 20^{-1-}$ mouse livers clearly revealed marked protection against hepatic steatosis (Figure 2, G-I). 
A
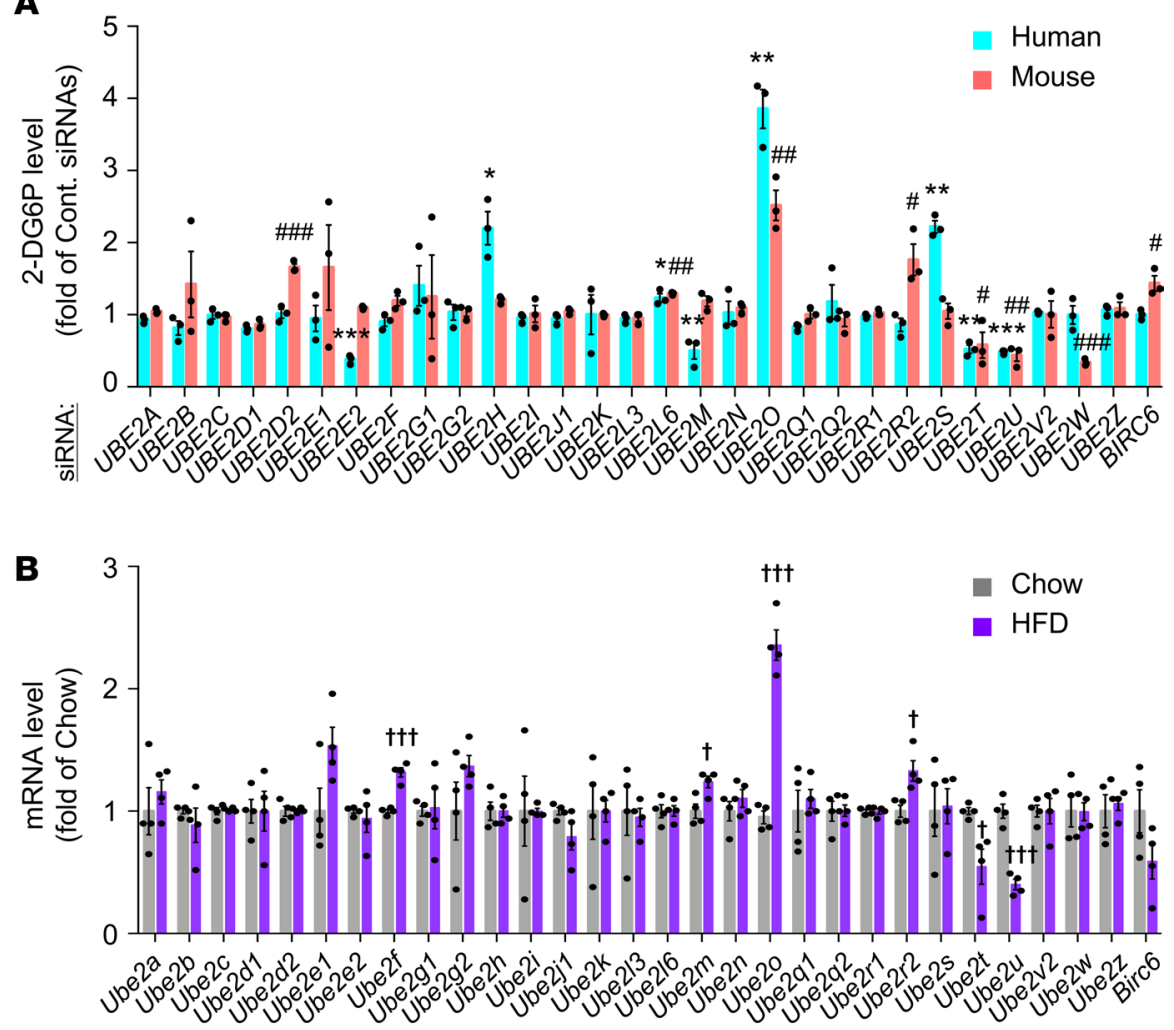

C

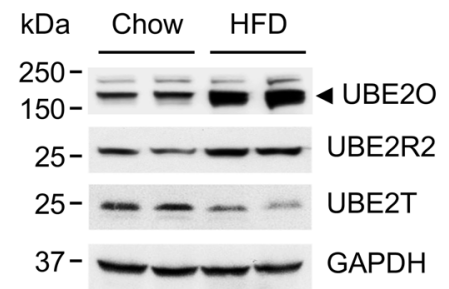

D

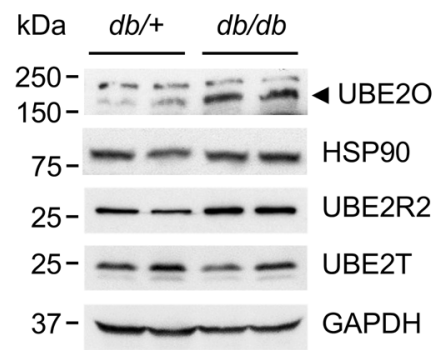

Figure 1. UBE2O is upregulated in obese subjects with type 2 diabetes. (A) An RNA interference (RNAi) screen identifies UBE2O as a potent regulator of glucose uptake in myotubes. Primary normal human skeletal myotubes (HSkMs) and C2C12 mouse myotubes expressing a nontargeting control siRNA pool (Cont. siRNAs) or a synthetic siRNA library targeting 30 human and mouse E2s, respectively, were subjected to a screen for insulin-stimulated 2-deoxyglucose uptake. 2-DG6P, 2-deoxyglucose-6-phosphate. $n=3$. (B) Total RNAs from quadriceps skeletal muscles of mice fed a normal chow or an HFD for 28 weeks were subjected to RT-qPCR. $n=4$. (C and D) Lysates from

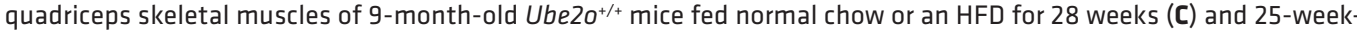
old type 2 diabetic $d b / d b$ and lean $(d b /+)$ mice (D) were subjected to immunoblotting. Error bars represent \pm SEM. $P$ value was determined by Student's $t$ test. ${ }^{*} P<0.05$, ${ }^{* *} P<0.01$, ${ }^{* *} P<0.001$, control siRNAs HSkMs vs. E2 siRNAs

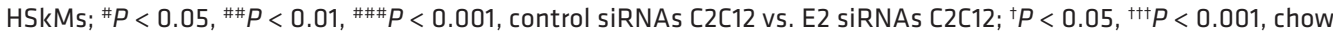
vs. HFD. Uncropped gels and blots are available in the supplemental material.

Alterations in body weight often reflect shifts in the balance between energy intake and expenditure (3). Strikingly, $\mathrm{Ube}^{-o^{--}}$mice showed higher oxygen consumption (Figure 2J and Supplemental Figure 1D) and a lower respiratory exchange ratio (RER) (Figure $2 \mathrm{~K}$ ) and presented higher energy expenditure normalized by lean mass during both the light and dark phases (Figure 2L and Supplemental Figure 1E), without significant accompanying changes in locomotor activity (Figure 2M). Furthermore, loss of Ube2o increased baseline thermogenesis, as determined by rectal temperature (Figure $2 \mathrm{~N}$ and Supplemental Figure 1F), and this increase was corroborated by a significant rise in the expression of genes involved in thermogenesis, such as Ucp1, Cidea, Dio2, and Prdm16 in brown adipose tissue (BAT) (Supplemental Figure 1G). The oxidative capacity of skeletal muscle also correlates with energy expenditure $(3,31)$, and $U b e 20^{-1-}$ mouse analyses of 
A

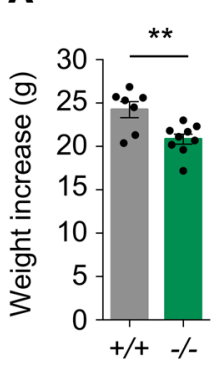

B

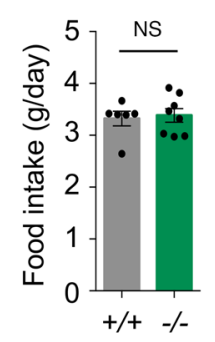

C

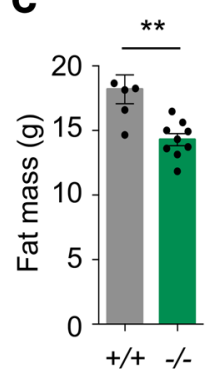

D

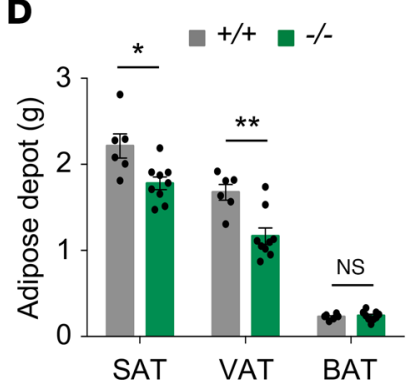

E

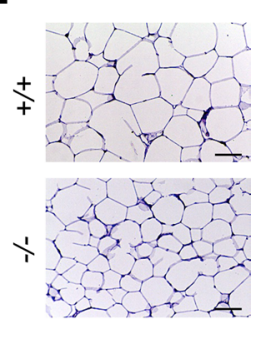

F

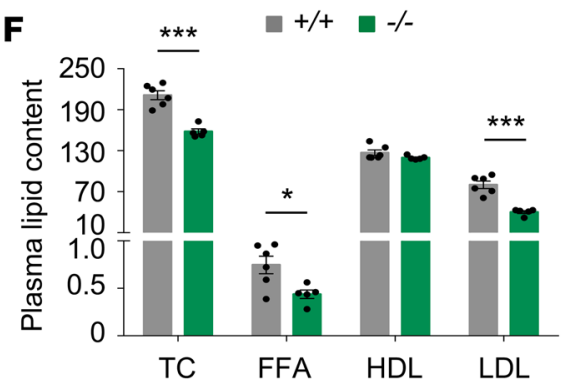

G

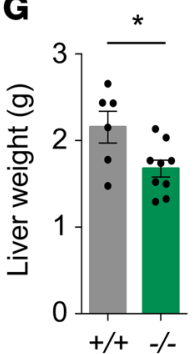

H

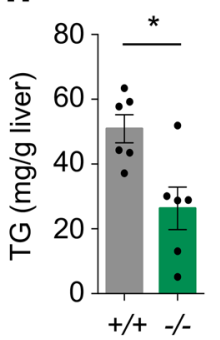

I

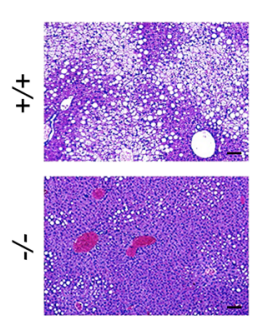

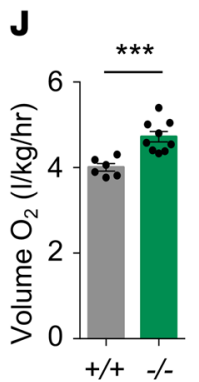
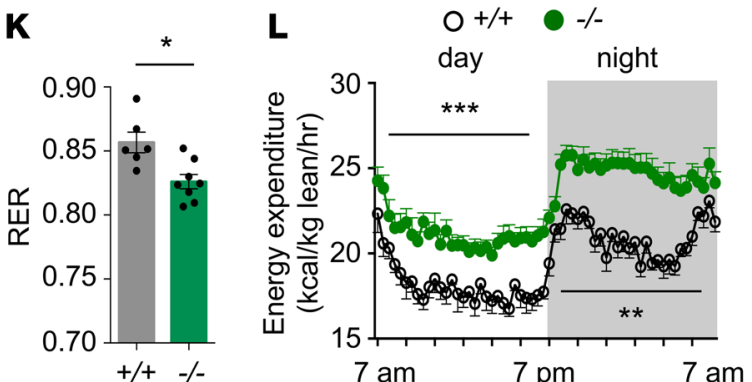

M

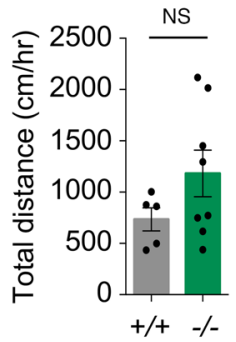

$\mathbf{N}$

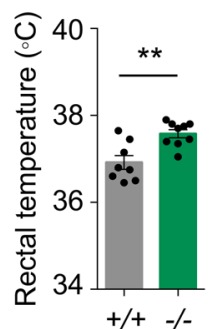

Figure 2. Ube2o ablation protects mice against diet-induced obesity and metabolic syndrome. (A) Body weight increase of $U b e 20^{+/+}$and $U b e 20^{-/-}$mice

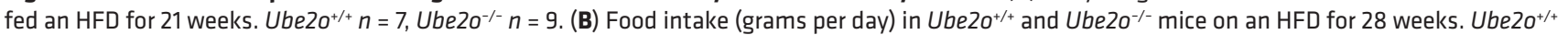

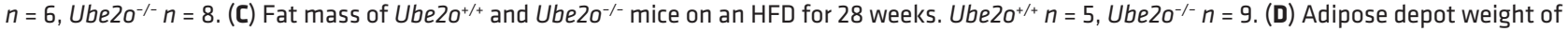
Ube $20^{+/+}$and Ube $20^{-/-}$mice on an HFD for 28 weeks. SAT, subcutaneous adipose tissue; VAT, visceral adipose tissue; BAT, brown adipose tissue. Ube20 ${ }^{+/+}$

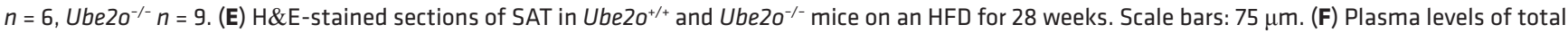

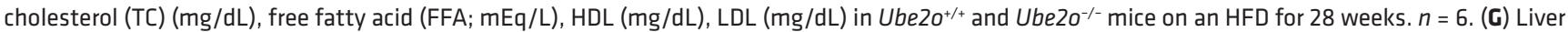

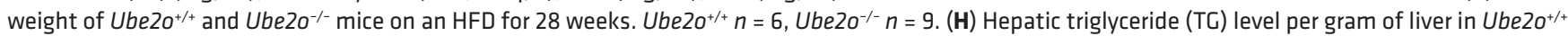

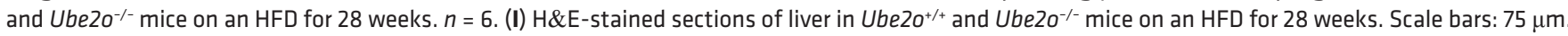

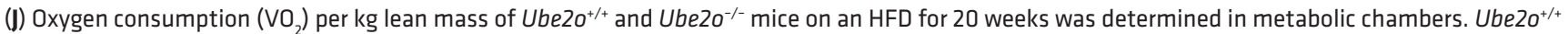

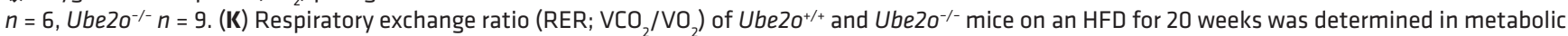
chambers. Ube $20^{+/+} n=6, U b e 20^{-/-} n=9$. (L) Energy expenditure during day and night per kg of lean mass of $U b e 20^{+/+}$and $U b e 20^{-/-}$mice on an $\mathrm{HFD}$ for 20 weeks was determined in metabolic chambers. Ube2 $0^{+/+} n=6, U b e 20^{-/-} n=9$. (M) Home cage locomotor activity during both the light and dark phases of $\mathrm{Ube} \mathrm{O}^{+/+}$and $\mathrm{Ube} 2 \mathrm{O}^{-/-}$mice on an HFD for 20 weeks was determined by using an automated combined indirect calorimetry system. $\mathrm{Ube} 2 \mathrm{O}^{+/+} n=5, \mathrm{Ube} 2 \mathrm{O}^{-1-}$

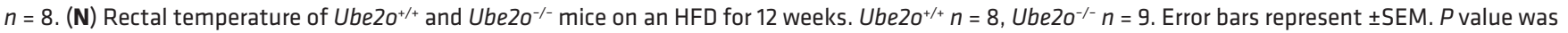
determined by Student's $t$ test $\left({ }^{*} P<0.05,{ }^{* *} P<0.01,{ }^{* *} P<0.001\right)$.

expression profiles of the transcription factor Ppargcla and its target genes - which are involved in mitochondrial biogenesis and oxidative metabolism and of mitochondrial activities such as succinate dehydrogenase (SDH) and citrate synthase (CS) - revealed a significant increase in the oxidative capacity of skeletal muscle (Supplemental Figure 1, H-J). Taken together, these results indicate that global knockout of Ube2o protects mice against HFD-triggered metabolic disorders such as obesity, dyslipidemia, and hepatosteatosis, and this effect appears to be associated with higher metabolic rates and energy expenditure.

Ube20-- mice preserve insulin sensitivity in diet-induced type 2 diabetes mouse model. As insulin resistance is a major risk factor for the development of diabetes and metabolic syndrome (1), we next explored whether loss of Ube2o could contribute to insulin sensitization. During dietary intervention, basal glucose lev-

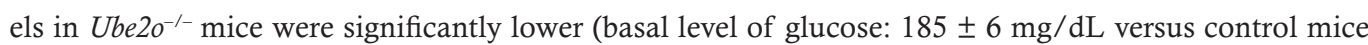
$217 \pm 9 \mathrm{mg} / \mathrm{dL}$ ) (Figure 3A), and glucose clearance rates were greatly improved (Figure 3B) compared 
A

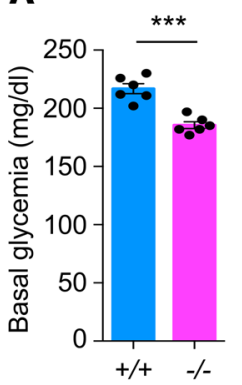

B

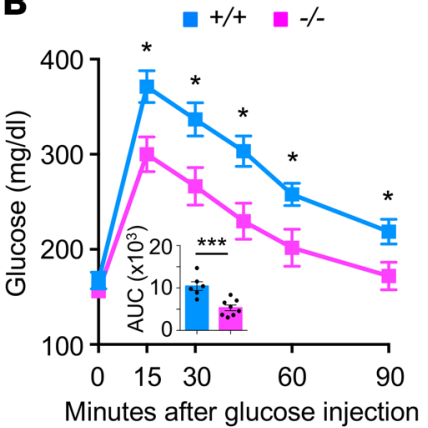

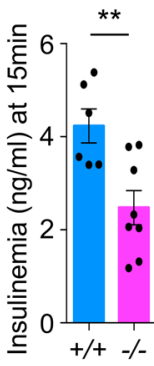

E

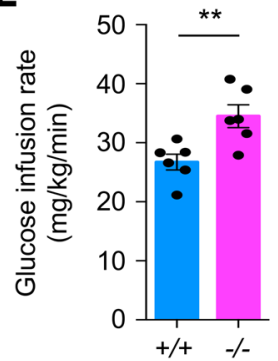

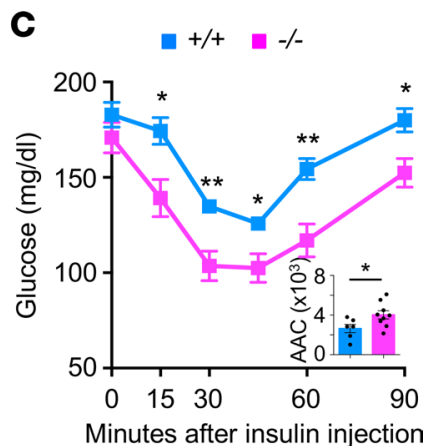

$\mathbf{F}$

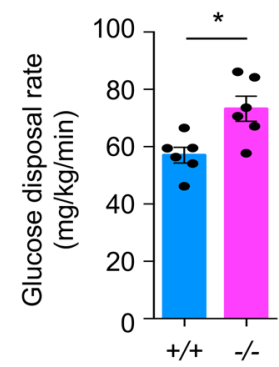

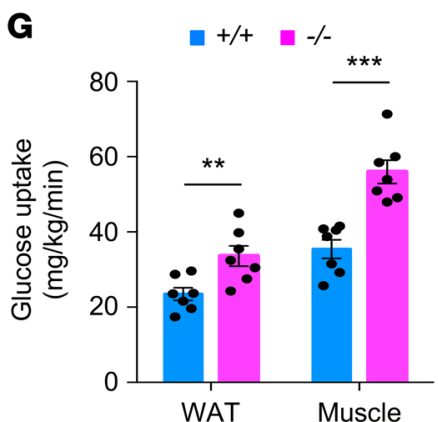
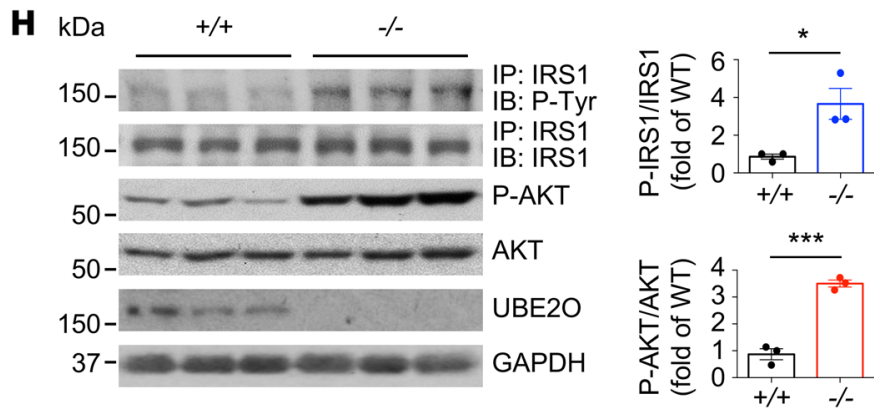

Figure 3. Ube $20^{-/-}$mice show improved insulin sensitivity in diet-induced type 2 diabetes mouse model. (A) Blood

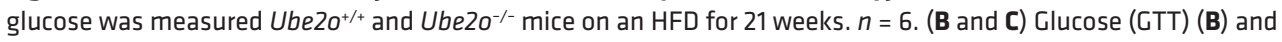

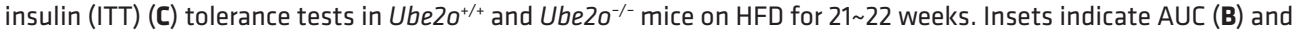
area above the curve (AAC) (C). Insulinemia at 15 minutes after the injection of glucose during a GTT test is shown in

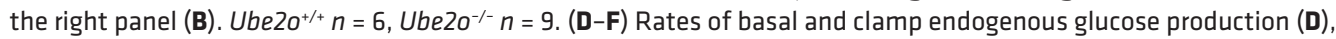
glucose infusion (E), and glucose disposal rate (F) during a hyperinsulinemic-euglycemic clamp study in Ube20 $\mathrm{O}^{+/+}$and Ube20 $0^{-1-}$ mice. $n=6$. (C) Insulin-stimulated ${ }^{14} \mathrm{C}$-2-deoxyglucose uptake was assessed in visceral WAT and gastrocnemius muscle of $\mathrm{Ube} \mathrm{O}^{+/+}$and $\mathrm{Ube2 \textrm {O } ^ { - / - }}$ mice during the final 45 minutes of the hyperinsulinemic-euglycemic clamps. $n=7$. (H) Immunoblots (left) and statistical data (right) showing insulin-induced (200 nM) tyrosine phosphorylation of IRS1 immunoprecipitates and $\mathrm{S} 473$ phosphorylation of AKT protein and their total protein levels in the extensor digitorum longus muscle from $U b e 20^{+/+}$and $U b e 20^{-/-}$mice on an HFD. $n=3$. Error bars represent $\pm=$ SEM. $P$ value was determined by Student's $t$ test $\left({ }^{*} P<0.05,{ }^{*} P<0.01,{ }^{* * *} P<0.001\right)$.

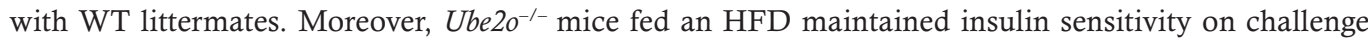
with intraperitoneal injection of insulin (Figure 3C). It is worth noting that the haplodeficiency of Ube2o in mice also resulted in the persistence of lower glucose levels, higher tolerance of glucose, and hypersensitivity to insulin in response to overnutrition (Supplemental Figure 2).

To further evaluate the mechanisms of glucose reduction and insulin sensitivity resulting from loss of Ube2o, we performed systemic glucose metabolism studies using a hyperinsulinemic-euglycemic clamp. Ablation of Ube2o in mice did not significantly alter endogenous glucose production under basal and clamped conditions (Figure 3D), but it significantly increased the glucose infusion rate (GIR) (Figure 3E) and the glucose disposal rate (GDR) (Figure 3F), indicating that loss of Ube2o promotes insulin action in vivo. Furthermore, insulin-stimulated glucose uptake assessed during the clamp using ${ }^{14} \mathrm{C}$-2-deoxyglucose was far greater in WAT and skeletal muscle from $\mathrm{Ube}_{2} \mathrm{o}^{-1}$ mice than from littermate controls (Figure 3G), 
suggesting that the glucose reduction and glucose disposal phenotypes observed in $U b e 20^{-/-}$mice were mainly due to increased glucose uptake in adipose and skeletal muscle, with no impact on hepatic glucose output. Increased tyrosine phosphorylation of insulin receptor substrate 1 (IRS1) and increased phosphorylation of Ser473 in AKT stimulated with insulin were found in the skeletal muscle of $U b e 20^{-/-}$mice on an HFD (Figure 3H), which was consistent with the data obtained from experiments with hyperinsulinemic-euglycemic clamps. Collectively, these results indicate that Ube2o ablation protects mice against HFD-induced hyperglycemia and insulin resistance.

Ablation of Ube2o in mouse skeletal muscle blocks diet-induced insulin resistance and metabolic decline. Expression of UBE2O occurs preferentially in metabolic tissues such as skeletal muscle, fat, and liver (28), but its tissue-specific roles in the regulation of metabolic homeostasis are so far undefined. As multiple metabolic organ systems are affected by whole-animal Ube2o knockout, we sought to elucidate the contributions of $\mathrm{UBE} 2 \mathrm{O}$ to insulin sensitization and energy homeostasis in the specific contexts of skeletal muscle, adipose tissue, and liver. We first utilized the Cre-loxP system to generate conditional knockout of Ube2o in mice. A floxed Ube2o allele was created in embryonic stem (ES) cells of the C57BL/ 6 mouse strain by introducing a loxP site into the introns flanking Ube2o exons 16 18 (encoding its catalytic domain), using a Neo cassette flanked by Frt sites as a selectable marker $\left(U b e 2 o^{f-n e o}\right)$ (Supplemental Figure 3A). The targeted ES cells were injected into blastocysts of C57BL/6 mice to obtain chimeric floxed mice. After germline transmission, the mice were crossed with mice expressing FLP recombinase to remove the Neo cassette (Ube2d ${ }^{f /+}$ ) (Supplemental Figure 3B). To knock out Ube2o, homozygous Ube2 $d^{f l f l}$ mice were crossed with transgenic mice [Tg(human alpha-skeletal actin [HSA]-cre)79Jme/J, Tg(adiponectin [Adipoq]-cre)1Evdr/J or Tg(serum albumin [Alb]-cre)21Mgn/J (32-34) expressing Cre recombinase under control of skeletal muscle-, adipose-, or liver-specific gene promoter (hereafter referred to as $U b e 2 o^{4 M u s}$, Ube $20^{4 A d i p}$, or $U b e 2 o^{4 L i v}$ ). UBE2O proteins were then found to be absent in skeletal muscle or adipose tissue, but not in other tissues, of Ube2 $0^{4 M u s}$ mice or Ube2 $o^{4 A d i p}$ mice, respectively (Supplemental Figure 3C).

When challenged with the HFD for 20 weeks, Ube $20^{4 M u s}$ mice gained significantly less body weight and fat mass relative to control mice, despite equivalent food intake (Figure 4, A-C, and Supplemental Figure 4, A-C). Sections of WAT further revealed a marked reduction in adipocyte size of Ube20 $0^{\Delta M u s}$ mice (Figure 4D). Plasma lipid profiles revealed reductions in TC and LDL levels and elevation of HDL levels in Ube $20^{4 M u s}$ mice on an HFD (Figure 4E). Furthermore, liver weight and hepatic triglyceride measurements as well as histological analysis of $U b e 2 o^{4 M u s}$ mouse livers clearly demonstrated marked and significant protection against HFD-induced hepatosteatosis (Figure 4, F-H). Notably, in Ube20 $0^{4 M u s}$ mice we observed lower expression of lipogenesis genes in WAT, higher expression of oxidative metabolism genes in skeletal muscle, and increased expression of thermogenesis genes in BAT (Figure 4I). Ube20 ${ }^{4 M u s}$ mice also showed increased oxygen consumption, energy expenditure normalized by lean mass (although the effects of muscle-specific Ube2o knockout appeared to be smaller than those of its global knockout), and baseline thermogenesis, with no significant alterations in spontaneous activity (Figure 4, J-N). Together, these results indicate that Ube2o deficiency in skeletal muscle exerts robust protective effects against HFD-induced metabolic disturbance, which further supports a causal relationship between skeletal muscle UBE2O upregulation and HFD-induced metabolic syndrome.

In order to track insulin sensitivity in relation to the development of metabolic syndrome, we performed glucose tolerance tests (GTTs) and insulin tolerance tests (ITTs) on Ube20 $0^{4 M u s}$ mice at various time points during the administration of an HFD. Glucose intolerance and insulin resistance in WT mice commenced as early as 5 6 weeks (Figure $4, \mathrm{O}$ and $\mathrm{P}$ ), even though obesity onset began at 8 10 weeks (Figure 4A). As the HFD challenge continued, both glucose intolerance and insulin resistance were progressively exacerbated in WT mice. In contrast, Ube20 $0^{4 M u s}$ mice exhibited enhanced glucose tolerance and insulin sensitivity even before the onset of appreciable obesity, and they remained sensitive to insulin signaling, which could likely be attributed to increased phosphorylation rates of IRS1 and AKT in skeletal muscle, but not in adipose tissue or liver (Figure 4Q and Supplemental Figure 4, D and E). Together with the profound protective effects of Ube2o ablation in skeletal muscle against metabolic disorders, these data strongly suggest that UBE2O-mediated muscle insulin resistance is necessary to instigate the pathogenic process.

Adipose- or liver-specific knockout of Ube2o has no beneficial effect on metabolic control or insulin sensitivity in HFD mice. Next, we investigated the adipose- or liver-specific roles of UBE2O in the control of metabolic

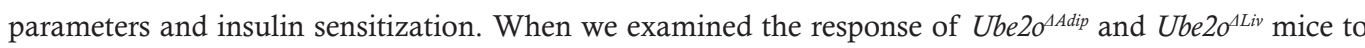
the HFD challenge, we found that weight accumulation in both was similar to that observed in control mice 
A

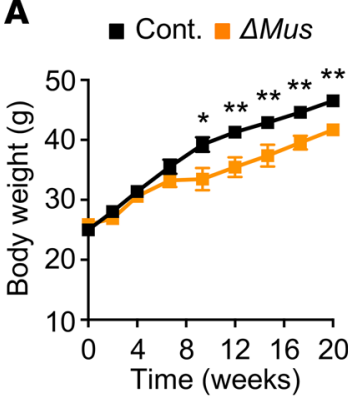

B

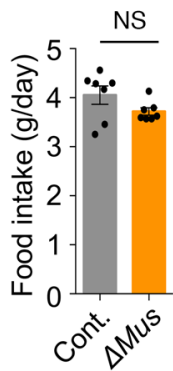

C

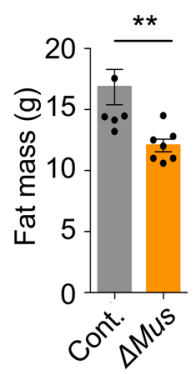

D

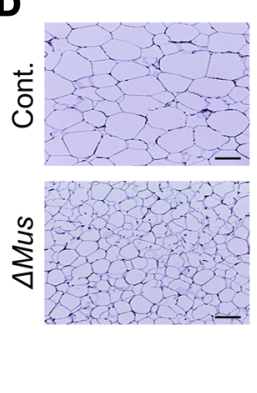

E

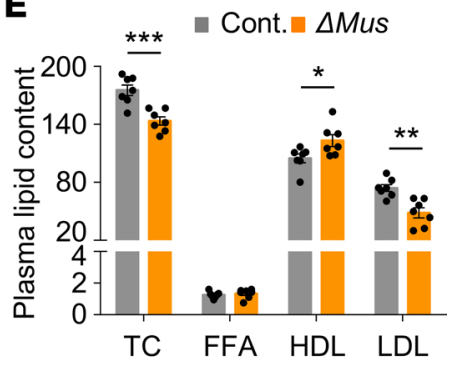

$\mathbf{F}$

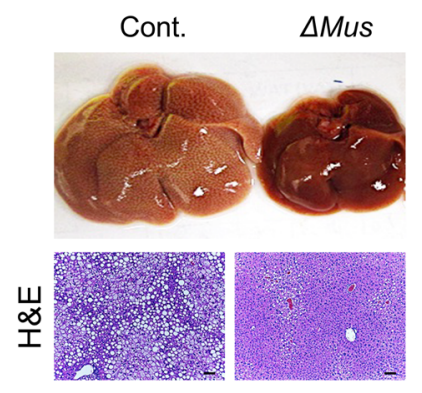

J

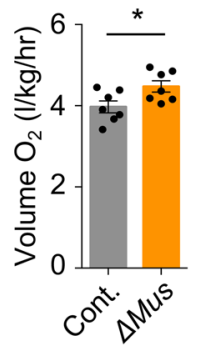

K

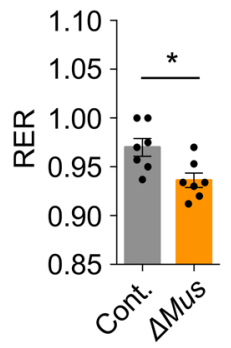

0

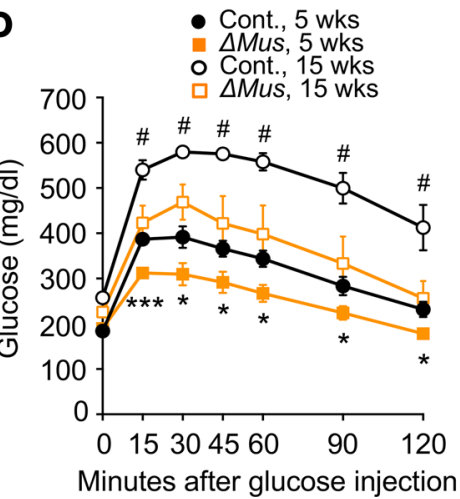

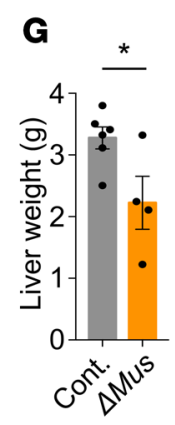

H
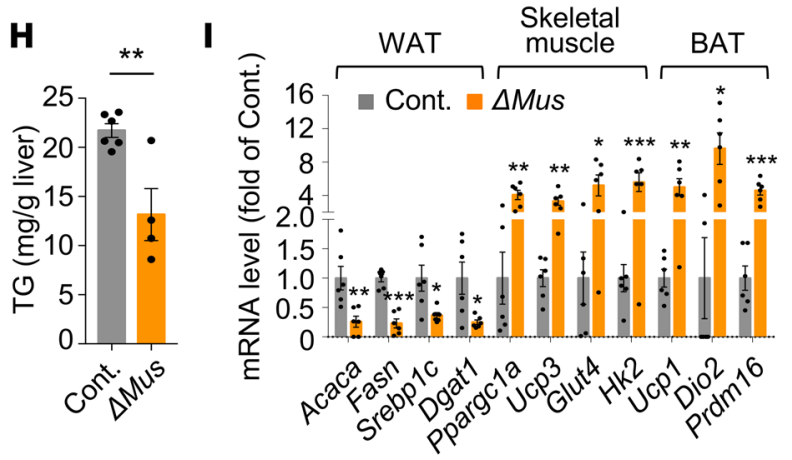

$\mathbf{L}$
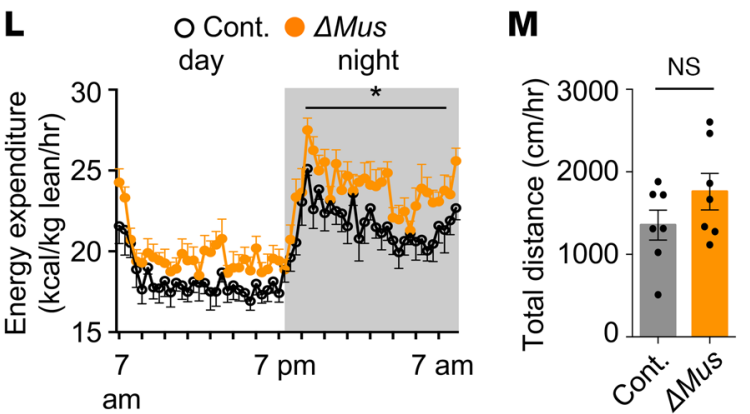

$\mathbf{N}$

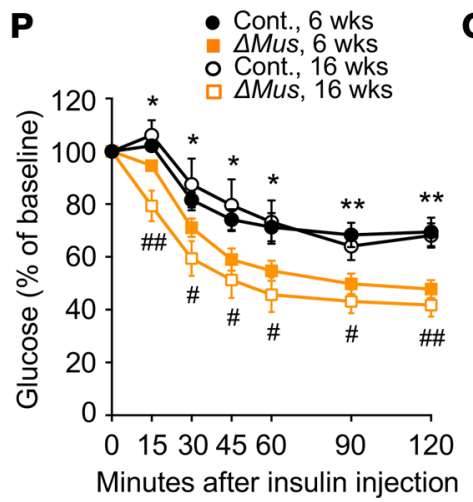

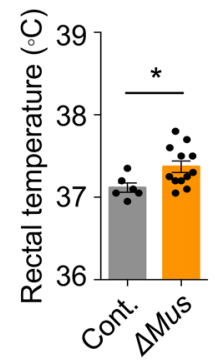

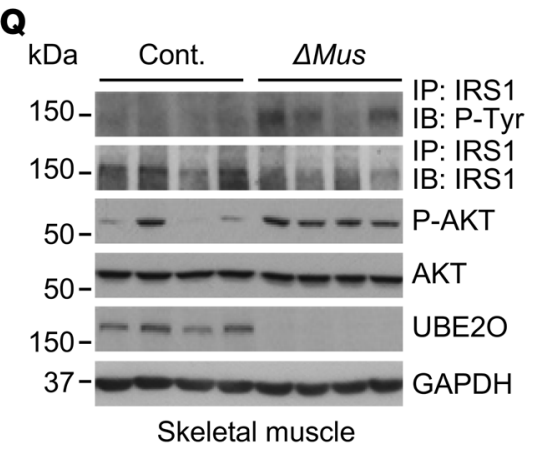

Figure 4. Muscle-specific ablation of Ube2o blocks diet-induced insulin resistance and metabolic disorders. (A) Body weights of control (Ube2 $\left.0^{f / / f}\right)$

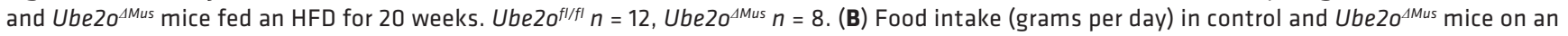
HFD for 16 weeks. $n=7$. (C) Fat mass of control and Ube20 ${ }^{4 M u s}$ mice on HFD for 20 weeks. $n=7$. (D) H\&E-stained sections of subcutaneous adipose tissue in control and Ube2o ${ }^{4 M u s}$ mice on an HFD for 20 weeks. Scale bars: $75 \mu \mathrm{m}$. (E) Plasma levels of TC (mg/dL), FFA (mEq/L), HDL (mg/dL), and LDL (mg/dL) in control and Ube2 ${ }^{4 M u s}$ mice on an HFD for 20 weeks. $n=7$. (F) Photographs and H\&E-stained sections of liver in control and

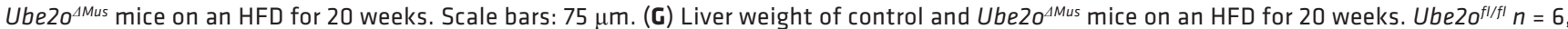

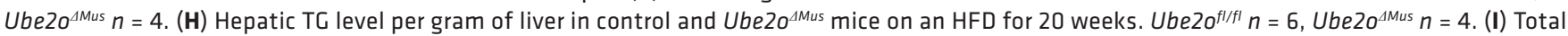
RNAs from WAT, skeletal muscle, and BAT of control and Ube2 $0^{4 M u s}$ mice on an HFD for 20 weeks were subjected to RT-qPCR. $n=6$. (J) VO, per kg of

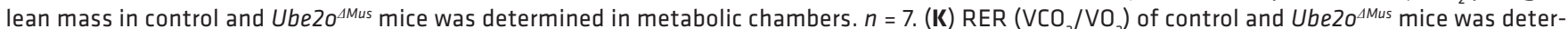
mined in metabolic chambers. $n=7$. (L) Energy expenditure during day and night per $\mathrm{kg}$ of lean mass of control and Ube20 $0^{4 M u s}$ mice was determined in metabolic chambers. $n=7$. (M) Home cage locomotor activity during both the light and dark phases of control and Ube20 ${ }^{\Delta M u s}$ mice was determined 
by using an automated combined indirect calorimetry system. $n=7$. (N) Rectal temperature of control and Ube20 ${ }^{4 M u s}$ mice on an HFD for 12 weeks. Ube2o ${ }^{f / / f l} n=6$, Ube20 $0^{4 M u s} n=12$. GTTs $(\mathbf{O})$ and ITTs $(\mathbf{P})$ in control and Ube20 $0^{4 \mathrm{Mus}}$ mice on an HFD for the indicated time periods. $n=7 .{ }^{*} P<0.05,{ }^{* *} P<$ $0.01,{ }^{* * *} P<0.001$, control 5 and 6 weeks HFD vs. Ube2 $20^{4 M u s} 5$ and 6 weeks HFD; ${ }^{*} P<0.05,{ }^{\# \#} P<0.01$, control 15 and 16 weeks HFD vs. Ube20 $0^{4 M u s} 15$ and 16 weeks HFD. (Q) Immunoblots showing insulin-induced (200 nM) tyrosine phosphorylation of IRS1 immunoprecipitates and S473 phosphorylation of AKT protein and their total protein levels in the extensor digitorum longus muscle from control and Ube20 ${ }^{4 M u s}$ mice on an HFD for 6 weeks. Error bars represent \pm SEM. $P$ value was determined by Student's $t$ test $\left({ }^{*} P<0.05,{ }^{* *} P<0.01,{ }^{* * *} P<0.001\right)$.

undergoing overnutrition (Figure 5A and Supplemental Figure 5A). The major fat tissues, including visceral fat, abdominal subcutaneous fat and brown fat, exhibited no obvious differences in weight in Ube $20^{4 A d i p}$ and $U b e 2 o^{4 L i v}$ mice compared with control mice (Figure $5 \mathrm{~B}$ and data not shown). Likewise, there were no alterations in adipocyte size (Figure 5C), nor did we observe any differences in plasma lipid response as determined by cholesterol levels (Figure 5D and Supplemental Figure 5B). Furthermore, adipose- or liver-specific knockout of Ube2o had no effect in HFD mice on hepatic steatosis (Figure 5, E-G, and Supplemental Figure 5, C and D).

We also attempted to examine whether loss of Ube2o in mouse adipose or liver tissue could contribute to systemic insulin sensitivity. In contrast to our findings in $U b e 20^{4 M u s}$ mice, basal glucose levels were unchanged, and glucose clearance rates were not improved in Ube2 $\sigma^{\Delta A d i p}$ mice and Ube2 $\sigma^{4 L i v}$ mice relative to control mice (Figure 5H and Supplemental Figure 5, E and F). Similarly, these mice did not perform significantly better in terms of either insulin sensitivity or insulin signaling (Figure 5, I and J, and Supplemental Figure 5, G and H). Collectively, these results indicate that adipose- or liver-specific knockout of Ube2o does not change the susceptibility of mice to diet-induced insulin resistance and metabolic syndrome.

Skeletal muscle UBE2O activates ubiquitination of AMPK 2 . Next, we sought to decipher the molecular mechanisms underlying UBE2O-mediated insulin resistance and metabolic disturbance in skeletal muscle. Although we recently identified UBE2O as an oncogene upstream of the AMPK pathway (16), current knowledge of the physiological interactions between UBE2O and AMPK in noncancerous contexts is very limited. Purification of endogenous UBE2O or AMPK $\alpha 2$ immunocomplexes from Ube2o-proficient or -deficient mouse skeletal muscle revealed a strong interaction between UBE2O and AMPK $\alpha 2$ in skeletal muscle (Figure 6A). In this context, UBE2O acted as an E2/E3 hybrid ubiquitin ligase, and directly ubiquitinated AMPK $\alpha 2$ in the presence of $\mathrm{E} 1$ enzyme alone, while AMPK $\alpha 2$ ubiquitination was significantly diminished by the catalytically inactive (C1037S [CS]) UBE2O mutant (Figure 6, B and C). Ubiquitination generally targets proteins for degradation. UBE2O, but not its ligase-defective mutant, indeed induced degradation of AMPK $\alpha 2$ in a proteasome-dependent manner (Figure 6D), and cycloheximide treatment revealed that UBE2O-mediated AMPK $\alpha 2$ ubiquitination greatly alters its protein turnover rate (Figure $6 \mathrm{E}$ ). In Ube2o-deficient mouse skeletal muscle tissues, the levels of endogenous AMPK $\alpha 2$ (but not AMPK $\alpha 1$ ) protein rose strikingly (Figure $6 \mathrm{~F}$ ), suggesting that skeletal muscle UBE2O binds to, ubiquitinates, and degrades AMPKa2.

To study the contribution of AMPK $\alpha 2$ to UBE2O-dependent glucose homeostasis, we performed overexpression or depletion of UBE2O in cultured C2C12 myotubes. Ectopic expression of WT (but not CS mutant) UBE2O inhibited insulin-stimulated glucose uptake, and suppressed AMPKa2 expression and phosphorylation of its substrates ACC and TBC1D1 (Figure 6, G and H), whereas knockdown of UBE2O resulted in increased glucose uptake accompanied by elevated levels of AMPK $\alpha 2$ expression and ACC and TBC1D1 phosphorylation (Figure 6, I and J). Critically, expression of Prkaa2 shRNAs reversed glucose uptake in $\mathrm{C} 2 \mathrm{C} 12$ cells depleted of UBE2O, suggesting that AMPK $\alpha 2$ ubiquitination and degradation by UBE2O inhibits insulin-induced glucose uptake in skeletal muscle cells.

AMPK is also known to regulate lipid metabolism through promotion of fatty acid oxidation (8), which prompted us to test whether the effect of UBE2O on fatty acid oxidation occurs through AMP$\mathrm{K} \alpha 2$. After administration of $\left[1-{ }^{14} \mathrm{C}\right]$-labeled palmitate, UBE2O-silenced $\mathrm{C} 2 \mathrm{C} 12$ cells showed much higher levels of palmitate oxidation through mitochondrial $\beta$-oxidation (as abrogated by treatment with etomoxir) as well as oxygen consumption (Supplemental Figure 6, A and B). Notably, downregulation of AMPK $\alpha 2$ by RNA interference ablated the promotion of fatty acid oxidation and oxygen consumption from palmitate in these cells, suggesting that AMPK $\alpha 2$ is very likely to be a major factor in reliable UBE2O function in fatty acid metabolism. As AMPK has been shown to be critical for mitochondrial biogenesis in skeletal muscle through the regulation of transcription via key transcription factors such as PGC1 $\alpha$ (35), we also evaluated the impact of UBE2O on PGC1 $\alpha$-mediated transcription. We found that 


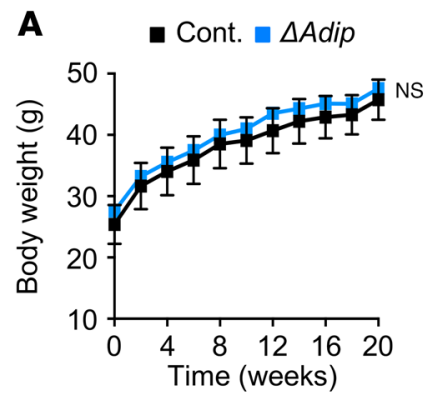

D

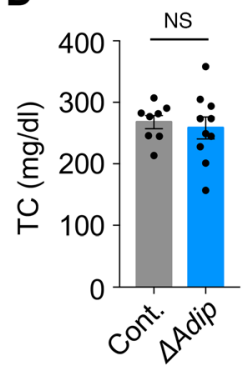

B

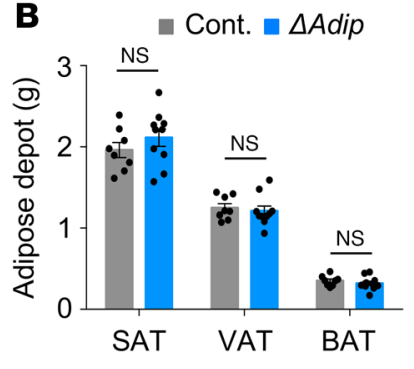

$\mathbf{F}$

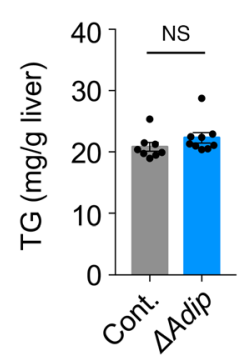

C

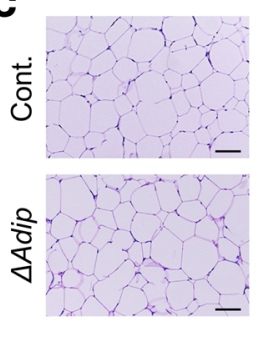

G

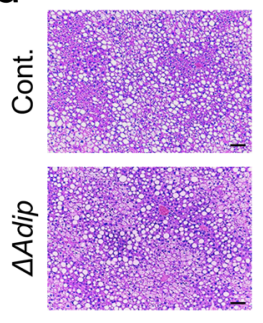

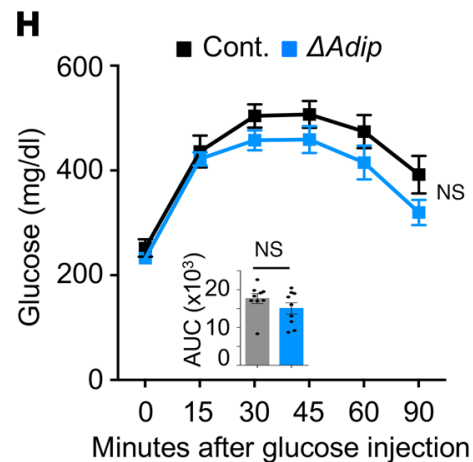
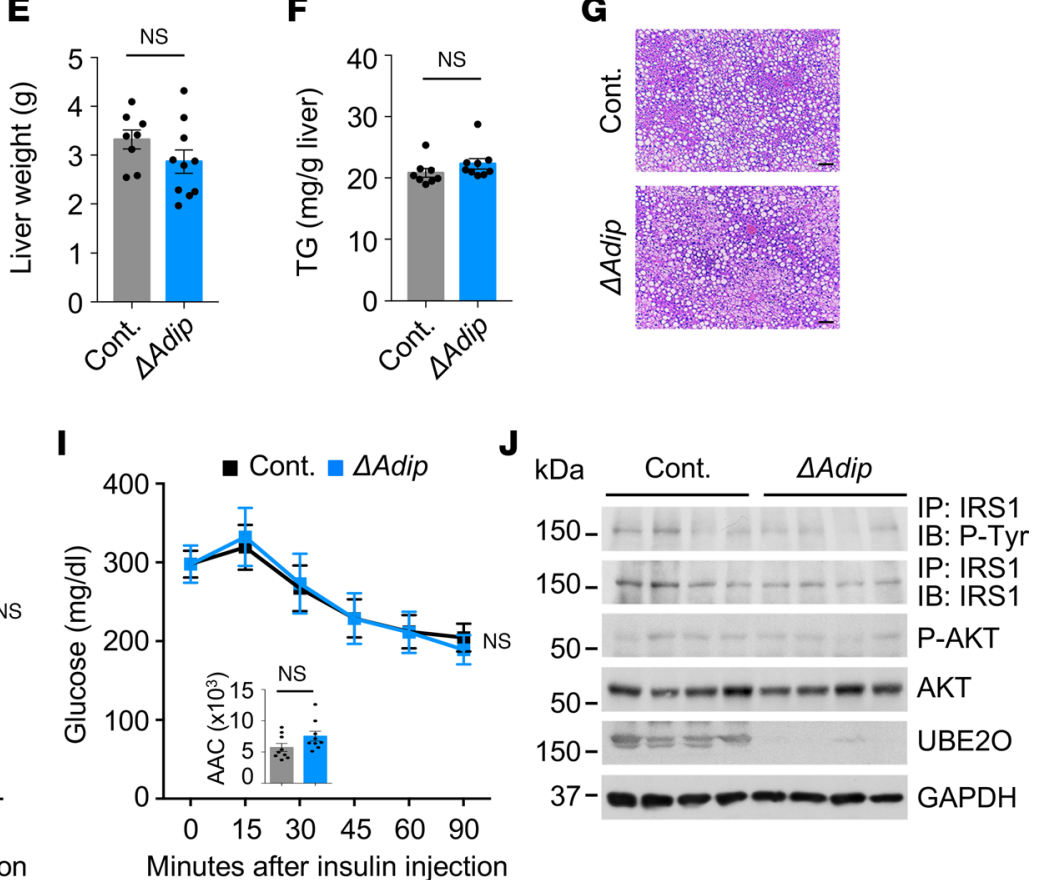

Figure 5. Adipose tissue-specific Ube2o-knockout mice do not have improved fat metabolism on an HFD. (A) Body weights of control (Ube2 $0^{f / f} f$ ) and Ube20 $0^{\text {AAdip }}$ mice fed an HFD for 20 weeks. $n=10$. (B) Adipose depot weight of control and Ube20 ${ }^{\text {AAdip }}$ mice on an HFD for 20 weeks. SAT, subcutaneous adipose

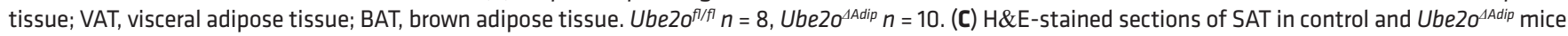
on an HFD for 20 weeks. Scale bars: $75 \mu \mathrm{m}$. (D) Plasma levels of TC (mg/dL) in control and Ube2 $0^{\text {AAdip }}$ mice on an HFD for 20 weeks. Ube20 $0^{f / f l} n=8, U b e 20^{4 A d i p}$ $n=10$. (E) Liver weight of control and Ube2 $0^{4 A d i p}$ mice on an HFD for 20 weeks. Ube2o $0^{f / f l} n=8$, Ube2 $0^{4 A d i p} n=10$. (F) Hepatic TC level per gram of liver in control

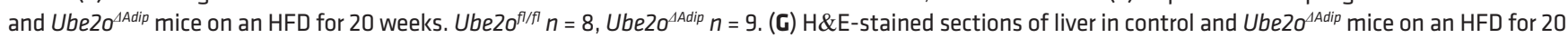
weeks. Scale bars: $75 \mu \mathrm{m}$. GTTs (H) and ITTs (I) in control and Ube20 ${ }^{\text {AAdip }}$ mice on an HFD for 16 weeks. Insets indicate AUC (H) and AAC (I). $n=9$. (J) Immunoblots showing insulin-induced (200 nM) tyrosine phosphorylation of IRS1 immunoprecipitates and S473 phosphorylation of AKT protein and their total protein levels in VAT from control and Ube20 $0^{\text {AAdip }}$ mice on an HFD for 20 weeks. Error bars represent \pm SEM. $P$ value was determined by Student's $t$ test.

the expression levels of Ppargcla and its target genes involved in mitochondrial biogenesis and oxidative metabolism were significantly elevated in UBE2O-depleted cells (Supplemental Figure 6C). This effect was reversed, however, by concomitant knockdown of AMPK $\alpha 2$, suggesting that AMPK 22 plays an essential role in UBE2O regulation of mitochondrial biogenesis. During energy stress, AMPK activation may promote metabolic adaptation by increasing NADPH generation in fatty acid oxidation and by decreasing NADPH consumption in fatty acid synthesis to eliminate ROS (36). Indeed, ectopic expression of UBE2O yielded a rapid depletion of NADPH and reduced glutathione (GSH) during energy stress, and increased ROS levels (Supplemental Figure 6, D-F). Conversely, loss of Ube2o resulted in marked reductions in the $\mathrm{NADP}^{+} / \mathrm{NADPH}$ ratio and the oxidized glutathione (GSSG)/GSH ratio, thus suppressing the accumulation of ROS in an AMPKa2-dependent manner (Supplemental Figure 6, G-I), suggesting that UBE2O regulates metabolic adaptation to energy stress through AMPK $\alpha 2$.

Activation of skeletal muscle AMPK 2 is both necessary and sufficient to mediate the protective effects of UBE2O ablation against diabetes and metabolic disorders. Next, we examined whether the mechanism for UBE2O ablation-induced protection against metabolic decline and insulin resistance during overnutrition specifically operates through the 
A

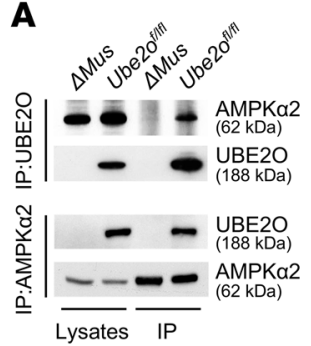

B

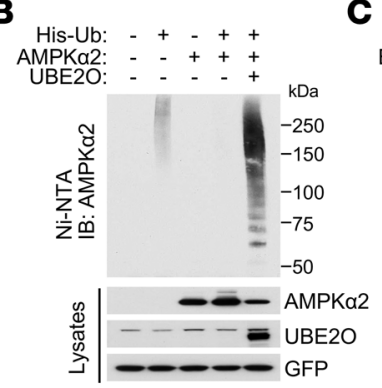

C AMPKa2: ++++
E1/ATP/Ub: -+++ UBE2O: - - WTCS $k$ Da
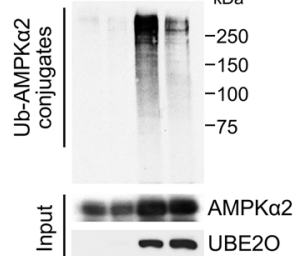

D

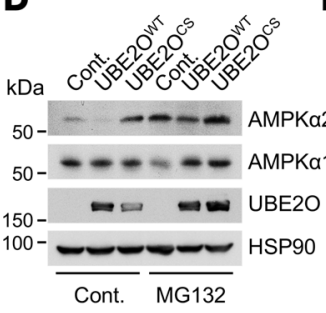

E

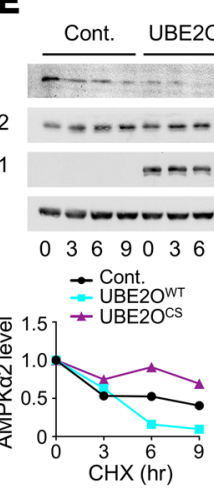

H

G

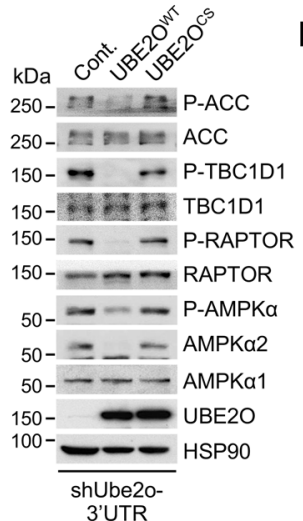

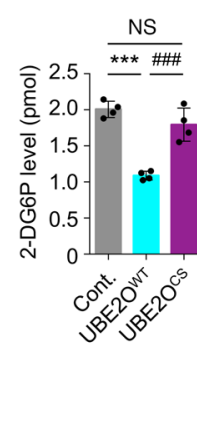

$\mathbf{F}$

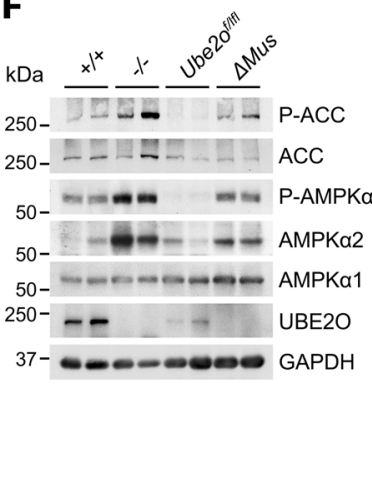

I

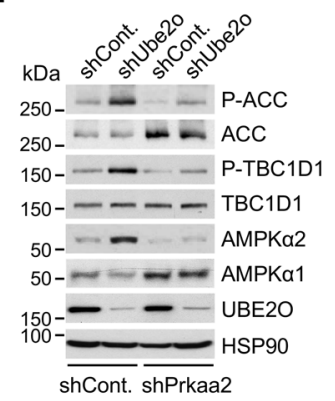

J

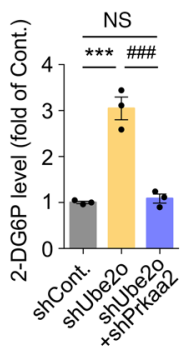

Figure 6. Regulation of AMPK $\alpha 2$ by skeletal muscle UBE20. (A) UBE2O (top) or AMPK $\alpha 2$ (bottom) immunoprecipitates of lysates from skeletal muscle of control (Ube2 $\left.0^{f / f f}\right)$ and Ube20 $0^{4 M u s}$ mice were subjected to immunoblotting for AMPK $\alpha 2$ or UBE2O. (B) Lysates from C2C12 myotubes transfected with the indicated plasmids were subjected to metal affinity purification for His-tagged ubiquitin (His-Ub), then immunoblotting for ubiquitinated AMPK $\alpha 2$. $\mathrm{Ni}-\mathrm{NTA}, \mathrm{Ni}^{2+}$-nitrilotriacetic acid. (C) Recombinant AMPK $\alpha 2$ proteins were subjected to in vitro ubiquitination assay in the presence of in vitro translated WT or C1037S (CS) mutant UBE20. (D) Lysates from C2C12 myotubes expressing WT or CS mutant UBE20 treated with MG132 $(10 \mu \mathrm{M})$ for 6 hours were subjected to immunoblotting. (E) Lysates from C2C12 myotubes expressing WT or CS mutant UBE2O treated with cycloheximide (CHX) for the indicated times were subjected to immunoblotting (top). AMPK $\alpha 2$ or AMPK $\alpha 1$ protein levels were quantified by normalizing to the intensity of the HSP9O band (bottom). (F) Lysates from skeletal muscle of 12-week-old Ube $2 \mathrm{O}^{+/+}$, Ube $2 \mathrm{O}^{-/-}$, $U b e 20^{f / f f}$, and Ube20 $0^{\Delta M u s}$ mice were subjected to immunoblotting for the indicated proteins. (C) Lysates from C2C12 myotubes expressing UBE20 shRNA together with exogenous WT or CS mutant UBE20 from a Ube20 ORF transcript lacking the 3' UTR sequence targeted by shRNA (shUbe2o-3'UTR) were subjected to immunoblotting for the indicated proteins. (H) Insulin-stimulated glucose uptake rate of C2C12 myotubes from G. 2-DG6P, 2-deoxyglucose-6-phosphate. $n=4$. (I) Lysates from C2C12 myotubes expressing Ube2o shRNA together with Prkaa2 shRNA were subjected to immunoblotting for the indicated proteins. (J) Insulin-stimulated glucose uptake rate of C2C12 myotubes from I. $n=3$. Error bars represent \pm SEM. $P$ value was determined by ANOVA $\left({ }^{* *} P<0.001,{ }^{\# \#} P<0.001\right)$.

AMPKa2 axis in vivo. Completely knocking out the AMPKa2 protein in a variety of tissues is likely to cause metabolic dysfunction in any mouse model (9), and thus we used heterozygous knockout of skeletal muscle Prkaa2 to test its interaction with Ube2o knockout's protection from HFD-induced metabolic decline and insulin resistance. For this purpose, we established skeletal muscle-specific Ube2o-homozygous and Prkaa2-heterozygous

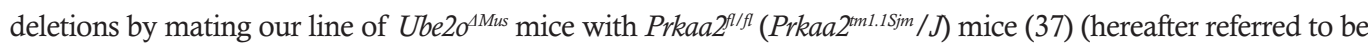


A

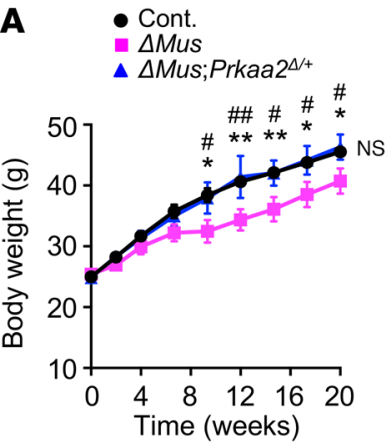

E

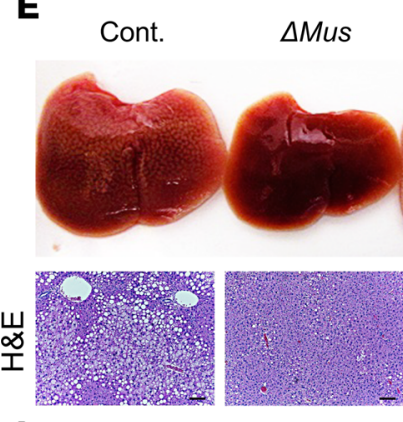

I

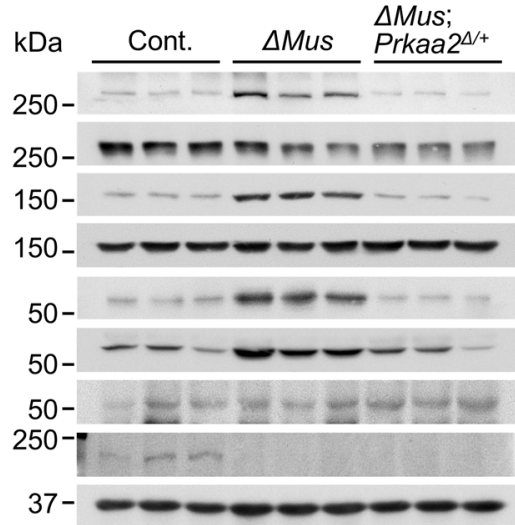

B $\quad$ Cont.

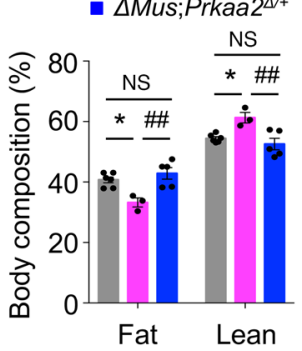

C

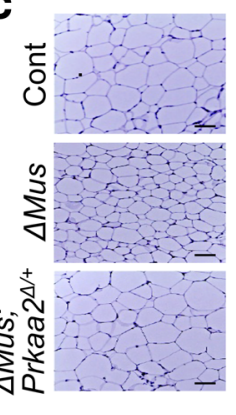

D

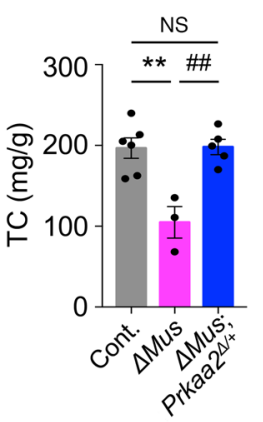

G

F

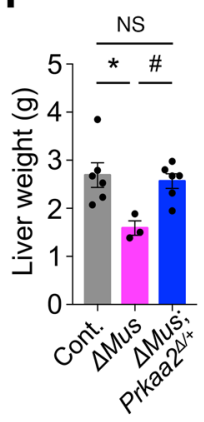

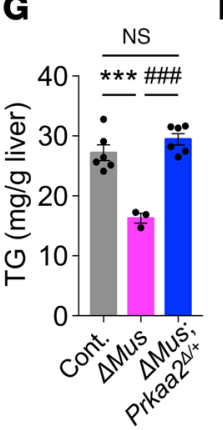

H

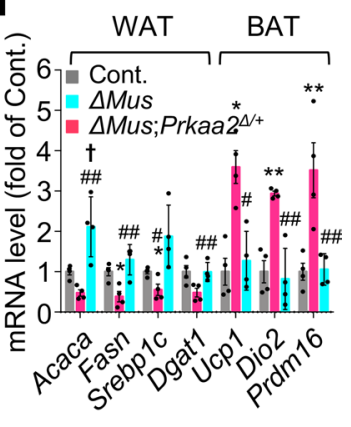

P-ACC

J ACC

P-TBC1D1

TBC1D1

P-AMPKa

AMPKa2

AMPKa1

UBE2O

GAPDH

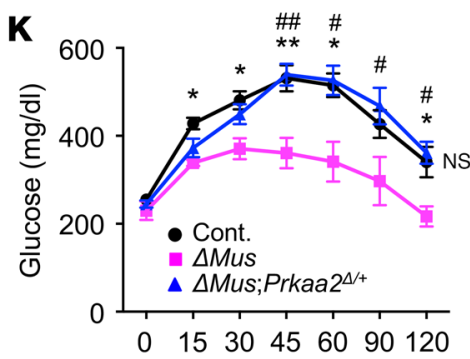

Minutes after glucose injection

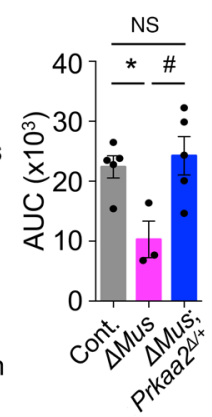

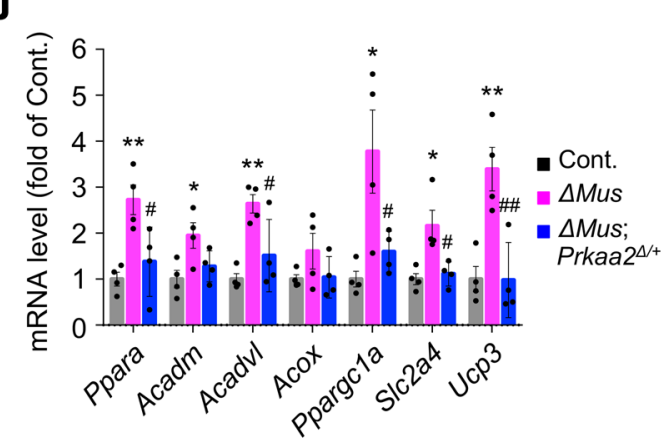

$\mathbf{L}$

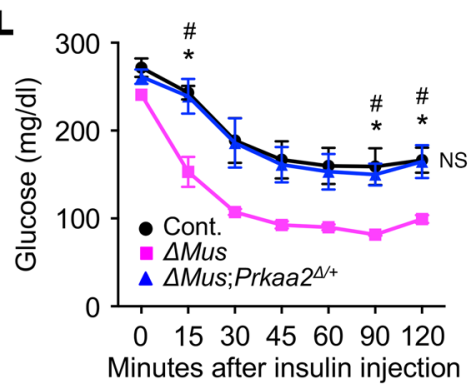

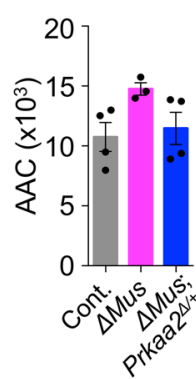

Figure 7. Activation of skeletal muscle AMPK $\alpha 2$ drives the protective effects of UBE2O loss on diabetes and metabolic disorders. (A) Body weights of control

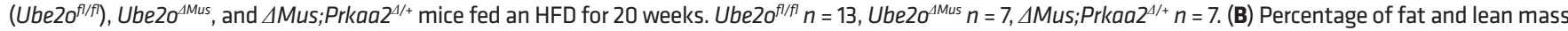

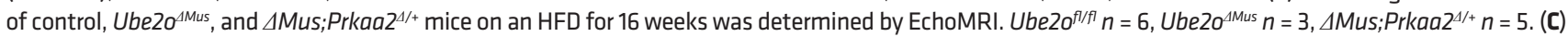

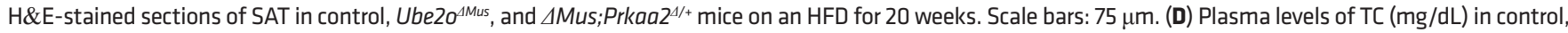

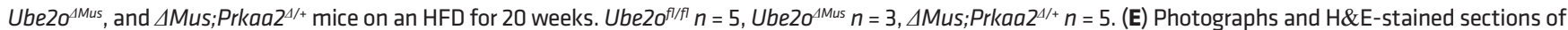

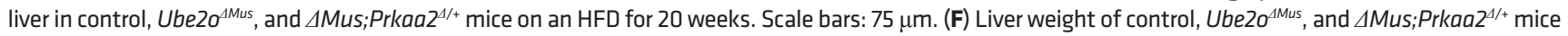

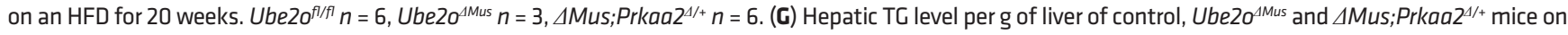

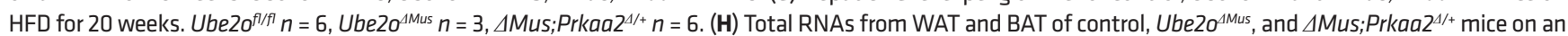
HFD for 20 weeks were subjected to RT-qPCR. $n=4$. (I) Lysates from skeletal muscle of control, Ube20 ${ }^{4 M u s}$, and $\triangle M$ Mus; Prkaa $2^{4 /+}$ mice on an HFD for 20 weeks were subjected to immunoblotting for the indicated proteins. (J) Total RNAs from skeletal muscle of control, Ube20 ${ }^{4 M u s}$, and $\triangle M u s ; P r k a a 2^{1 /+}$ mice on an HFD for 20

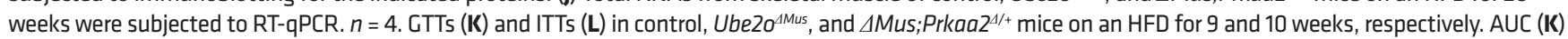
and $\mathrm{AAC}(\mathrm{L})$ are shown in the right panels. Ube2 $0^{f / f} n=5$, Ube2 $0^{4 \mathrm{Mus}} n=3, \Delta \mathrm{Mus} ; \operatorname{Prkaa}^{4 /+} n=5$. Error bars represent \pm SEM. $P$ value was determined by ANOVA. ${ }^{*} P$

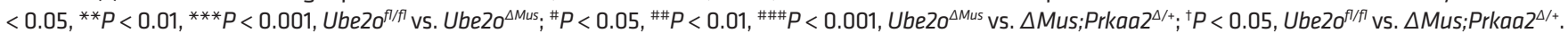


as $\triangle M u s ; P r k a a 2^{4 /+}$; all animals shared the same C57BL/6 background). Changes in their body weight and metabolic parameters during dietary intervention were then tracked. Consistent with our earlier results, Ube20 1 Mus mice gained significantly less weight and fat mass upon HFD feeding. In contrast, $\triangle M u s ; P r k a a 2^{4 /+}$ mice showed gains in body weight and adiposity similar to those of control mice (Figure 7, A-C). After 20 weeks on an HFD, serum cholesterol levels in $\triangle M u s ; P r k a a 2^{4 /+}$ mice were also largely comparable to those of control mice (Figure 7D). Furthermore, heterozygous deficiency of Prkaa2 in skeletal muscle abolished the protective effects of Ube2o ablation on HFD-induced hepatic steatosis, as evidenced by enlarged livers, histologic appearance of vacuoles in liver, and elevated triglyceride levels (Figure 7, E-G). Likewise, Prkaa2-heterozygous deletion reversed the expression signature of genes involved in fatty acid and triglyceride synthesis or thermogenesis seen in $U b e 20^{4 M u}$ mice (Figure $7 \mathrm{H})$. Taken together, these results suggest that skeletal muscle AMPK $\alpha 2$ is a key downstream factor for UBE2O-controlled obesity and metabolic syndrome in HFD mice.

We also assessed the contribution of AMPK $\alpha 2$ to UBE2O-mediated regulation of glucose homeostasis and lipid metabolism in skeletal muscle. Notably, muscle glucose uptake (via increased TBC1D1 phosphorylation) and fatty acid oxidation (via phosphorylation and inactivation of ACC) were clearly impaired in $\triangle M u s ; P r k a a 2^{4 /+}$ mice relative to Ube20 $0^{\Lambda M u s}$ mice (Figure 7I). Also, heterozygous knockout of skeletal muscle Prkaa2 ablated increased expression of genes involved in fatty acid oxidation and mitochondrial biogenesis (through the transcription factor PGC1 $\alpha$ ) in the skeletal muscle of Ube20 $0^{4 M u s}$ mice (Figure 7J). While Ube2 $20^{4 M u s}$ mice fed an HFD showed a much higher clearance of glucose and sensitivity to insulin, $\triangle M u s ; P r k a a 2^{4 /+}$ mice still exhibited both impaired glucose tolerance and insulin resistance in response to overnutrition (Figure 7, $\mathrm{K}$ and $\mathrm{L}$ ). Taken together, these results demonstrate the functional importance of UBE2O in the AMPK pathway and that muscular AMPK $\alpha 2$ activation is both necessary and sufficient to mediate the protective effects of $U B E 2 O$ loss against diet-induced insulin resistance and metabolic disorders.

\section{Discussion}

Our data demonstrate what we believe to be a previously unrecognized role for the ubiquitin-conjugating enzyme UBE2O in the regulation of energy homeostasis and insulin sensitivity in vivo. Whereas UBE2O is known to be recurrently amplified in human cancers (38-42), we have now shown that UBE2O is universally upregulated in animal models exhibiting insulin resistance and metabolic disorders. Whole-body knockout of Ube2o in mice reverses the gains in weight, white fat mass, and body lipid content resulting from overnutrition. The leanness of $U b e 20^{-1-}$ mice could account for the improved metabolism observed in adipose tissue, liver, and skeletal muscle. Elevated energy expenditure (or bioenergetics) due to more efficient metabolic mechanisms in the mitochondria of BAT and skeletal muscle $(3,43)$ could explain the reduced adiposity observed in $U b e 20^{-/-}$mice. Indeed, $U b e 2 o^{-/-}$mice display increased heat production and have more active BAT without alteration of its mass. Likewise, mitochondrial respiration and biogenesis are significantly higher in Ube2o-deficent BAT and skeletal muscle.

However, we cannot rule out the possibility that the BAT activation observed in $U b e 2 o^{-1-}$ mice could be due to hyperthyroidism or hyperadrenergic activity $(44,45)$. UBE2O has been previously implicated in the regulation of adipogenesis in vitro (26), so it is also possible that Ube2o ablation could result in a lean mouse with aberrant adipocyte differentiation. By characterizing the outcomes of inactivation of Ube2o in specific tissues, however, we have been able to show that Ube2o ablation is not beneficial in adipose tissue upon HFD feeding (see below). However, Ube2o global knockout results in increased insulin sensitivity, leading to glucose reduction, and enhanced glucose tolerance and whole-body glucose disposal. Likewise, glucose uptake and insulin signaling in skeletal muscle in vivo are UBE2O dependent.

Our studies with tissue-specific Ube2o-knockout lines provide robust genetic evidence that the protective effects of Ube2o ablation against diet-induced insulin resistance and metabolic disorders are associated with skeletal muscle rather than adipose or liver tissue. Specifically, skeletal muscle-specific ablation of Ube2o results in a higher metabolic rate, lower adiposity, reduced hepatosteatosis, and increased insulin sensitivity, replicating many of the features of whole-body knockout, while other mouse models involving fat and liver have not exhibited this phenotype. The interesting and important question raised by this study concerns the relationship between enhanced muscle insulin sensitivity and obesity resistance in $U b e 2 o^{\Delta M u s}$ mice. The increased energy expenditure observed in $U b e 20^{\Delta M u s}$ mice could result in decreased adipose stores, which in turn would lead to enhanced insulin sensitivity as a secondary effect (4). However, this seems rather unlikely, for the following reasons: (i) the improvement in insulin sensitivity induced by muscle-specific ablation of Ube2o occurs even before changes in adiposity 
in HFD-challenged mice; and (ii) the Ube2o haplodeficiency in mice preserves glucose reduction and higher sensitivity to insulin without significantly affecting obesity after 7 weeks of HFD. We thus favor a model whereby the increased insulin sensitivity observed in skeletal muscle of $U b e 20^{\Delta M u s}$ mice leads to a higher metabolic rate, which results in decreased adiposity. It is also possible that a non-cell-autonomous mechanism of muscle-specific ablation of Ube2o could lead to decreased white adipose mass. It would therefore be interesting to further determine the non-cell-autonomous role(s) of skeletal muscle UBE2O in controlling whole-body energy homeostasis.

Our findings from $\triangle M u s ; P r k a a 2^{4 /+}$ mice clearly demonstrate the functional importance of UBE2O in the AMPK pathway and implicate muscular AMPK $\alpha 2$ as a key mediator of its effects. The interaction of $\mathrm{UBE} 2 \mathrm{O}$ and $\mathrm{AMPK} \alpha 2$ is critical for the control of several cellular processes, including glucose transport via phosphorylation of TBC1D1 $(46,47)$; fatty acid oxidation via phosphorylation and inactivation of ACC $(48,49)$; and mitochondrial biogenesis through the transcription factor PGC1 $\alpha(35,50)$.

Given our previous work suggesting that AMPK $\alpha 2$ regulation of mTORC1 activity is essential for the oncogenic action of UBE2O in a cancerous context (16), it is possible that reduced mTORC1 signaling could afford metabolic protection in Ube2o-deficient skeletal muscle. mTORC1 activity is known to be crucial for muscle development, as modulations that blunt mTORC1 activity cause muscular dystrophy, while enhanced mTORC1 activity is required for muscle hypertrophy $(51,52)$. Neither Ube $20^{4 M u s}$ nor $\triangle$ Mus;Prkaa $2^{4 /+}$ mice, however, showed abnormalities at birth or muscle dystrophy in adulthood, indicating that the UBE2O/AMPK $\alpha 2$ axis is not essential for muscle development. It is possible that inhibition of mTORC1 signaling in Ube2o-deficient skeletal muscle could induce autophagy, which would in turn result in muscle atrophy $(53,54)$. However, Ube2 $0^{4 M u s}$ mice do not exhibit muscular atrophy; rather they exhibit higher soleus (but not gastrocnemius or tibialis anterior) muscle mass compared with control mice. Blunting of mTORC1 abrogates its feedback inhibition of insulin signaling and thereby strongly induces the PI3K/AKT pathway $(55,56)$. Thus, it will be interesting to see whether part of the phenotype of Ube2 $20^{4 M u s}$ mice could be based on the hyperactivation of PI3K/AKT elicited by the absence of the inhibitory feedback into insulin receptor substrate (IRS).

Ube $20^{4 M u s}$ mice had improved glucose homeostasis and lipid metabolism during dietary intervention, which is consistent with previous results in muscle-specific AMPK-active mice (57-59), and heterozygous knockout of skeletal muscle Prkaa2 ablated this phenotype. This finding also suggests that the remaining AMPK $\alpha 1$ could not fully compensate for the lack of AMPK $\alpha 2$ in $\triangle M u s ; P r k a a 2^{4 /+}$ mice. Although the functional significance of different AMPK isoforms remains unclear, AMPK $\alpha 1$ and AMPK $\alpha 2$ demonstrate some specificity in tissue distribution, subcellular localization, and substrate selection (60-63). In skeletal muscle, AMPK complexes containing the $\alpha 2$ catalytic subunit are predominant, whereas AMPK $\alpha 1$ is mainly expressed in adipose tissue. AMPK $\alpha 1$ - and AMPK $\alpha 2$-containing complexes each account for about half of total AMPK activity in rodent liver.

AMPK is activated in skeletal muscle by exercise, and this activation mediates the beneficial effects of exercise on diabetes and metabolic disorders $(64,65)$, which explains the rapid rise in research into pharmacological activators of muscle-specific AMPK as therapeutic drugs. Indeed, activation of skeletal muscle AMPK with the small molecule AMPK agonists MK-8722 and PF-739 has been shown to provide significant benefits in terms of glucose reduction, muscle glucose disposal, and mitochondrial biogenesis in rodents and nonhuman primates, independent of AMPK activation in other tissues $(66,67)$.

Our findings should prove relevant to the development of drugs designed to interfere with the interaction between UBE2O and AMPK $\alpha 2$ or inhibit UBE2O ubiquitin ligase activity for AMPK $\alpha 2$ activation. This strategy may represent a powerful means of ameliorating diabetes and metabolic syndrome by exploiting not only the activation of skeletal muscle AMPK but also its subsequent contributions to muscle insulin sensitivity, leading to improvements in systemic physiology and metabolic health.

\section{Methods}

Mice. To generate Ube2o-knockout mice, we injected 3 different fully verified Ube2 $2 o^{\operatorname{tml}(\text { KOMP)Mpb }}$ ES clones (JM8A3.N1), which were created by the CSD consortium (CSD81565) from the trans-NIH Knockout Mouse Project (KOMP), into blastocysts derived from C57BL/6 mice to produce chimeras. Transmitting chimeric mice were bred from EIIa-cre transgenic mice to generate the Ube2o- alleles. $\mathrm{Cre}^{+} ; \mathrm{Ube}^{-} \mathrm{O}^{+/-}$ males were backcrossed twice to C57BL/6 females, and progeny of these matings that were $\mathrm{Cre}^{-}$were then backcrossed to littermates to yield the experimental cohort. Mice were genotyped by PCR with the 
following primers: WT, 5'-CTTGGGATGCAGTGCAGATA-3', 5'-GACACCTACCTGGCACCATT-3'; KO, 5'-GCTACCATTACCAGTTGGTCTGGTGTC-3'， 5'-CATGACACTCCCTTTCAAGCATGG-3' . In order to create a tissue-specific Cre-mediated conditional knockout of Ube2o, Ube2 $o^{f l}$ mice (C57BL/6 background) were generated under contract by inGenious Targeting Laboratory. Briefly, a floxed Ube2o allele was created in ES cells of the C57BL/6 mouse strain by introducing a loxP site into the introns flanking Ube2o exons 16 18, using a Neo cassette flanked by Frt sites as a selectable marker. The targeted ES cells were injected into blastocysts of C57BL/6 mice to obtain chimeric floxed mice (Ube2o $o^{f-n e o}$ ). After germline transmission, the mice were crossed with mice expressing FLP recombinase to remove the Neo cassette $\left(U b e 2 o^{f /+}\right)$. To knock out Ube2o, homozygous $U b e 2 o^{f l / f}$ mice were crossed with transgenic mice $[\mathrm{Tg}$ (human alpha-skeletal actin (HSA)-cre)79Jme/J, Tg(adiponectin (Adipoq)-cre) $1 \mathrm{Evdr} / \mathrm{J}$ or Tg(serum albumin (Alb)-cre)21Mgn/J (32-34) expressing Cre recombinase under control of skeletal muscle-, adiposeor liver-specific gene promoter. Ube $20^{f l}$ mice were genotyped by PCR with the following primers: Ube $20^{f t}$, 5'-GCCATTGTTCACGTGGAGATGTGAAT-3', 5'-GTCGCTGTTGTCTGGATAATCACACTC-3'. Cre mice were genotyped by PCR with the following primers: cre, 5'-GCCGTCTGGCAGTAAAAACTATC-3', 5'-GTGAAACAGCATTGCTGTCACTT-3'. To test trans-heterozygous interaction between

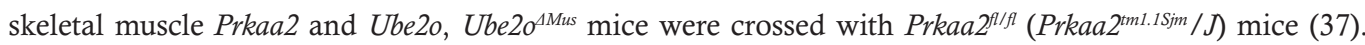
Prkaa $2^{f l}$ mice were genotyped by PCR with the following primers: Prkaa2 $2^{f}, 5^{\prime}$-GCAGGCGAATTTCTGAGTTC-3', 5'-TCCCCTTGAACAAGCATACC-3'. $d b / d b$ mice (male $d b / d b$ mice at 25 weeks of age) (68) and age-matched lean control mice $(d b /+)$ were from the Jackson Laboratory.

Mouse metabolic studies. Metabolic rates in mice were measured by indirect calorimetry using a Comprehensive Lab Animal Monitoring System (CLAMS, Columbus Instruments). The food intake was also monitored by the system. Mice were housed individually in metabolic chambers with free access to water and food for 3 days for acclimatization. Fat and lean mass of mice were then measured by EchoMRI (EchoMRI LLC), before animals were put back into metabolic chambers and monitored for the next 3 days for oxygen consumption $\left(\mathrm{VO}_{2}\right)$, carbon dioxide production $\left(\mathrm{VCO}_{2}\right)$, and food intake. Energy expenditure was calculated using the formula energy expenditure $=\left(3.815+1.232 \mathrm{VO}_{2} / \mathrm{VCO}_{2}\right) \times \mathrm{VO}_{2}$, and normalized to lean body mass. Body temperature was assessed in mice using a RET-3 rectal probe (Kent Scientific). ITTs and GTTs were performed as previously described (69). Briefly, mice were fasted for 6 hours with free access to drinking water. For ITTs, insulin was administered intraperitoneally $(0.4 \mathrm{mU} / \mathrm{g}$ of mice fed normal chow or $0.8 \mathrm{mU} / \mathrm{g}$ of mice fed an HFD), and blood glucose was measured at various times after injection from the tip of the tail with a Glucometer (Accu-Chek, Roche). For GTTs, D-glucose was administered intraperitoneally ( $2 \mathrm{~g} / \mathrm{kg}$ of mice on chow or $1 \mathrm{~g} / \mathrm{kg}$ of mice on an HFD), and blood glucose levels were monitored. At 15 minutes after glucose injection, blood was also collected for insulin quantitation. To analyze blood lipids, mouse blood was collected using the submandibular pouch technique. Sera were separated using serum separator tubes (catalog 365956, BD Biosciences). Analysis of lipid profile was performed in sera collected from mice that were fasted for 6 hours. Triglyceride, FFA, and cholesterol tests were analyzed at the Mouse Metabolism Core, Diabetes Research Center, Baylor College of Medicine, Houston, Texas, USA. Serum insulin concentrations were determined by the ELISA kit (Mouse Ultrasensitive Insulin ELISA, ALPCO Diagnostics). To measure hepatic triglyceride levels, mouse liver $(100 \mathrm{mg}$ ) was extracted using the Folch method with slight modifications, and triglyceride concentrations were then determined by the triglyceride colorimetric assay kit (MilliporeSigma).

Hyperinsulinemic-euglycemic clamp. Hyperinsulinemic-euglycemic clamp studies were performed in unrestrained mice using regular human insulin (Humulin $\mathrm{R}, 4 \mathrm{mU} / \mathrm{kg}$ body weight) in combination with HPLC-purified $\left[3-{ }^{3} \mathrm{H}\right]$ glucose and $\left[{ }^{14} \mathrm{C}\right] 2$-deoxyglucose. In brief, a microcatheter was inserted into the jugular vein by survival surgery, and mice were allowed to completely recover over 4-5 days. Studies were then performed in conscious mice. Overnight-fasted conscious mice received a priming dose of HPLC-purified $\left[3-{ }^{3} \mathrm{H}\right]$ glucose $(10 \mu \mathrm{Ci})$ and then a constant infusion $(0.1 \mu \mathrm{Ci} / \mathrm{min})$ of labeled glucose for approximately 3.5-4.0 hours. Blood samples were collected from the tail vein at 0, 50, and 60 minutes to measure the basal glucose production rate. After approximately 1 hour of infusion, mice were primed with regular insulin (16 $\mathrm{mU} / \mathrm{kg}$ body weight as a bolus), followed by an approximately 2-hour constant insulin infusion (4 $\mathrm{mU} / \mathrm{kg} / \mathrm{min}$ ). Using a separate pump, 25\% glucose was used to maintain the blood glucose level at 100-140 mg/dL, as determined every 6-9 minutes using a glucometer (LifeScan).

Glucose uptake measurement. For mouse tissue-specific uptake, we injected $\left[1-{ }^{14} \mathrm{C}\right] 2$-deoxyglucose $(10$ $\mu \mathrm{Ci}$ ) as a bolus during hyperinsulinemic-euglycemic clamp at 45 minutes before the end of the clamps and collected blood samples at 5, 10, 15, 25, 35, and 45 minutes. Glucose uptake in different tissues was then 
calculated from a plasma ${ }^{14} \mathrm{C}$-2-deoxyglucose profile fitted with double exponential curve and tissue content of ${ }^{14} \mathrm{C}$-2-deoxyglucose-6-phosphate. To determine the rate of cellular glucose uptake in culture, the differentiation of primary normal human skeletal myoblasts (HSkMs; A11440, Gibco) and C2C12 mouse myoblasts was induced with insulin/1\% horse serum in medium following a standard protocol. Cells were then transfected with a siGENOME nontargeting siRNA pool (D-001206-13, Dharmacon) or 30 human or mouse E2 siGENOME SMARTpool siRNA library using the Dharmafect I siRNA transfection reagent and serum starved overnight. Cells were washed 3 times with PBS and subjected to glucose deprivation by preincubation with KRPH buffer (20 mM HEPES, $5 \mathrm{mM} \mathrm{KH}_{2} \mathrm{PO}_{4}, 1 \mathrm{mM} \mathrm{MgSO}_{4}, 1 \mathrm{mM} \mathrm{CaCl}, 136 \mathrm{mM}$ $\mathrm{NaCl}, 4.7 \mathrm{mM} \mathrm{KCl}$ [pH 7.4]) containing 2\% BSA for 40 minutes. Cells were then stimulated with or without $1 \mu \mathrm{M}$ insulin for 20 minutes to activate glucose transporter. Glucose uptake was initiated by addition of 1 $\mathrm{mM}$ 2-deoxyglucose for 20 minutes, before cells were lysed and intracellular glucose was measured using a Glucose Uptake Colorimetric Assay Kit (BioVision).

CS assay. $10 \mathrm{mg}$ tissue or $1 \times 10^{6}$ cells were lysed in $200 \mu \mathrm{L}$ of an extraction buffer $\left(100 \mathrm{mM} \mathrm{KH}_{2} \mathrm{PO}_{4} /\right.$ $\mathrm{Na}_{2} \mathrm{HPO}_{4}$ [pH 7.2], $2 \mathrm{mM}$ EDTA). The CS assay was carried out using $10 \mu \mathrm{L}$ of the lysates in a 96-well plate. CS activity was measured by adding $200 \mu \mathrm{L}$ of the reaction solution containing $0.12 \mathrm{mM}$ DTNB, $0.15 \mathrm{mM}$ acetyl-CoA, $0.6 \mathrm{mM}$ oxaloacetate, and $100 \mathrm{mM}$ Tris- $\mathrm{HCl}(\mathrm{pH}$ 7.4) to each well. Absorbance was measured at $415 \mathrm{~nm}$ every 12 seconds for 7 minutes using the kinetic mode of a microplate reader. CS activity was normalized to total protein content.

Immunohistochemistry. Mouse tissue samples were either fixed in 4\% paraformaldehyde solution in PBS before being embedded in paraffin or directly mounted in O.C.T. before being stored at $-80^{\circ} \mathrm{C}$. Tissue morphology was examined in H\&E-stained sections. Images were acquired with a Leica DM1000 microscope. The size of adipose cells was quantified using ImageJ (NIH) software. Succinate dehydrogenase (SDH) staining was performed as previously described (70). Briefly, $10-\mu \mathrm{m}$ cryosections of tibialis anterior were incubated at $37^{\circ} \mathrm{C}$ for 10 minutes in substrate buffer $(0.2 \mathrm{M}$ phosphate buffer containing sodium succinate $(25 \mathrm{mg} / \mathrm{mL})$ and NBT [1 mg/mL]). Sections were then washed 3 times with water, followed by 2 washes each with increasing and decreasing concentrations of acetone (30\%,60\%, and $90 \%)$. The stained slides were scanned on an Automated Cellular Image System III (Dako) for quantification by digital image analysis. Quantification of IHC staining density was performed by ImagePro software (Media Cybernetics) and calculated based on the average staining intensity and the percentage of positively stained cells.

Reagents and antibodies. 2-Deoxyglucose, dexamethasone, etomoxir, FCCP, D-glucose, insulin, 3-isobutyl-1-methyl-xanthine, oligomycin, pioglitazone, and rotenone were purchased from MilliporeSigma. $\left[1-{ }^{14} \mathrm{C}\right]$ palmitate and $\left[9,10(\mathrm{n}){ }^{3} \mathrm{H}\right]$ palmitic acid were purchased from PerkinElmer. HFD (45\% energy as fat, catalog D12451) was obtained from Research Diets. Antibodies against pS79-ACC (1:1000, 3661), ACC (1:1000 for immunoblotting, 3662), pS473-AKT (1:1000, 9271), pan-AKT (1:2000, 4691), IRS1 (1:500, 2390), TBC1D1 (1:1000, 5929), pS792-RAPTOR (1:1000, 2083), RAPTOR (1:500, 2280), pT172-AMPK $\alpha(1: 1000,2535)$, GAPDH (1:5000, 2118), and pTyr (PY100; 1:500, 9411) were from Cell Signaling Technology. Antibodies specific for AMPK $\alpha 2$ (1:1000, 07-363), pS237-TBC1D1 (1:500, 07-2268), and anti-IRS1 antibody conjugated with protein A agarose (catalog 16-146) were from Millipore. Anti-UBE2R2 (1:2000, 14077-1-AP) and -UBE2T $(1: 1000,10105-2-A P)$ antibodies were purchased from Proteintech. Antibodies against ubiquitin (1:1000, MMS258R), HA-Tag (1:2000, MMS-101R), and GFP (1:5000, MMS-118R) were from Covance. Anti-Flag (1:5000, F1804), -HSP90 (1:1000, H1775) and - $\beta$-Actin (1:5000, A1978) antibodies were purchased from MilliporeSigma. The UBE2O antibody (1:2000, A301-873A) was from Bethyl Laboratories; the AMPK $\alpha 1$ antibody (1:1000, NBP2-22127) was from Novus Biologicals; and the polyubiquitin antibody (1:1000, PW8805) was from Biomol.

Plasmids and RNA interference. Myc-tagged AMPK 22 (Addgene) was obtained by PCR and cloned into pGEX5X-1. The mutant construct (C1037S) of Flag-tagged mouse UBE2O (Origene) was generated by site-directed mutagenesis and cloned into pBabe retroviral vector. All constructs were verified by full-length sequencing. pLKO.1 lentiviral vectors encoding mouse Ube2o shRNAs (TRCN0000095039, TRCN0000095041) and mouse Prkaa2 shRNAs (TRCN0000360775, TRCN0000360846) were from MilliporeSigma. GFP-directed shRNA was used as control. The sources of 30 human or mouse E2 siRNA duplexes used are in shown Supplemental Tables 1 and 2.

Immunoprecipitation. Quadriceps skeletal muscles of $U b e 2 o^{f l f l}$ and $U b e 20^{\Delta M u s}$ mice were lysed in buffer containing 50 mM Tris- $\mathrm{HCl}$ (pH 7.5), $150 \mathrm{mM} \mathrm{NaCl}, 1 \mathrm{mM} \mathrm{MgCl}, 1 \mathrm{mM}$ EDTA, and 1\% Triton X-100 and cleared by centrifugation. Immunoprecipitation with anti-UBE2O or anti-AMPK $\alpha 2$ antibodies was performed as previously described (16). 
In vivo ubiquitination assay. Cells were lysed in buffer containing $6 \mathrm{M}$ guanidinium- $\mathrm{HCl}, 10 \mathrm{mM}$ Tris- $\mathrm{HCl}$ (pH8.0), $5 \mathrm{mM}$ imidazole, and $10 \mathrm{mM} \beta$-mercaptoethanol and cleared by centrifugation. His-ubiquitin-conjugated AMPK $\alpha 2$ was purified from cell lysates using the $\mathrm{Ni}^{2+}$-NTA spin column (QIAGEN) under denaturing conditions. The extent of ubiquitination was then analyzed by immunoblotting with anti-AMPK $\alpha 2$.

In vitro ubiquitination assay. The in vitro ubiquitin ligase activity of $\mathrm{UBE} 2 \mathrm{O}$ was analyzed as previously described (16). Briefly, in vitro translated WT or CS mutant UBE2O was incubated with GST-AMPK $\alpha 2$ in the presence of $50 \mu \mathrm{g} / \mathrm{mL}$ human E1 (E-305, Boston Biochem), $4 \mu \mathrm{g} / \mathrm{mL}$ ubiquitin aldehyde (Calbiochem), and ubiquitin (10 mg/mL) (MilliporeSigma), along with an ATP-regenerating system (7.5 mM creatine phosphate, $1 \mathrm{mM}$ ATP, $1 \mathrm{mM} \mathrm{MgCl}, 0.1 \mathrm{mM}$ EGTA, rabbit creatine phosphokinase type I [30 $\mathrm{U} / \mathrm{mL}$ ] (MilliporeSigma]) to a final reaction volume of $20 \mu \mathrm{L}$. Samples were incubated for 1 hour at room temperature, and the extent of ubiquitination of AMPK $\alpha 2$ was analyzed by immunoblotting.

$R T-q P C R$. Total RNA from tissues was extracted with Trizol reagent (Invitrogen) and reverse transcribed with PrimeScript reverse transcriptase (Clontech). Expression of specific mRNAs was determined with a LightCycler (Roche) using the SYBR green PCR master mix (Roche). Reactions were performed in triplicate, and relative amounts of CDNA were normalized to hypoxanthine guanine phosphoribosyl transferase (HPRT), Hsp90, or $18 S$ rRNA. RT-qPCR primer sequences are listed in Supplemental Table 3.

Fatty acid oxidation measurement. Cells were preincubated for 3 hours in medium containing $1 \mu \mathrm{Ci} / \mathrm{mL}$ $\left[1-{ }^{14} \mathrm{C}\right]$ palmitate and nonlabeled palmitate. Palmitate was coupled to fatty acid-free BSA at a molar ratio of 5:1. Following incubation, ${ }^{14} \mathrm{CO}_{2}$ and ${ }^{14} \mathrm{C}$-ASM (acid-soluble metabolites) were measured; the medium was transferred into a custom-made Teflon 48-well trapping plate. The plate was clamped and sealed, and perchloric acid was injected through the perforations in the lid into the medium, which drove $\mathrm{CO}_{2}$ through the tunnel into an adjacent well, where it was trapped in $1 \mathrm{~N} \mathrm{NaOH}$. Following trapping, the medium was spun twice, and ${ }^{14} \mathrm{C}$-ASM was measured by scintillation counting. Aliquots of $\mathrm{NaOH}$ and medium were transferred into scintillation vials, and radioactivity was then measured on a multipurpose scintillation counter (Tricarb 2100 TR, Packard). Data were normalized to total protein content. Alternatively, cells were incubated overnight in medium containing $100 \mu \mathrm{M}$ palmitate $(\mathrm{C} 16: 0)$ and $1 \mathrm{mM}$ carnitine. In the final 2 hours of incubation, the cells were pulsed with $1.7 \mu \mathrm{Ci}\left[9,10(\mathrm{n}){ }_{-}^{3} \mathrm{H}\right]$ palmitic acid, and the medium was collected to analyze the released ${ }^{3} \mathrm{H}_{2} \mathrm{O}$, formed during cellular oxidation of $\left[{ }^{3} \mathrm{H}\right]$ palmitate. In brief, the medium was TCA precipitated, and supernatants were neutralized with $\mathrm{NaOH}$ and loaded onto ion exchange columns packed with the DOWEX 1X2-400 resin (MilliporeSigma). The radioactive product was eluted with water and quantitated by a liquid scintillation counter (Beckman Coulter). Oxidation of $\left[{ }^{3} \mathrm{H}\right]$ palmitate was normalized to total protein content.

Oxygen consumption rate measurement. $1 \times 10^{4}$ cells were seeded into XF 96-well microplates (Seahorse Bioscience); 12 hours later, after replacing the medium with $180 \mu \mathrm{L}$ bicarbonate-free medium prewarmed at $37^{\circ} \mathrm{C}$, the cells were preincubated for 30 minutes before starting the assay procedure. Oxygen consumption rate (OCR) was then measured with a Seahorse XFe96 extracellular flux analyzer (Seahorse Bioscience). After 3 baseline measurements, cells were treated sequentially with $1 \mu \mathrm{M}$ oligomycin to inhibit ATP synthase for 3 measurements, $0.5 \mu \mathrm{M}$ FCCP to induce uncoupling of the cells for 3 measurements, and $1 \mu \mathrm{M}$ rotenone and $1 \mu \mathrm{M}$ antimycin $\mathrm{A}$ to inhibit complex I and III for 3 measurements. OCR was normalized to total protein content.

NADPH and GSH assays. Intracellular levels of NADPH and total NADP (NADPH plus NADP ${ }^{+}$) were measured using the NADP/NADPH quantitation colorimetric kit (BioVision) according to the manufacturer's instructions. In brief, $1 \times 10^{5}$ cells were seeded into 6-well plates; the next day, cells were lysed in $200 \mu \mathrm{L}$ extraction buffer and centrifuged. For NADPH extraction, supernatants were incubated at $60^{\circ} \mathrm{C}$ for 30 minutes; next, $98 \mu \mathrm{L}$ NADP-cycling buffer containing 1.3 U G6PD (2 $\mu \mathrm{L})$ was added to 96-well plates containing $50 \mu \mathrm{L}$ cell extracts. After a 5-minute incubation in the dark at room temperature, $10 \mu \mathrm{L}$ of 10-mM glucose 6-phosphate (G6P) was added to the mixture, and the change in absorbance at $450 \mathrm{~nm}$ was measured every 30 seconds for 4 minutes using a microplate reader. The concentration of $\mathrm{NADP}^{+}$was calculated by subtracting [NADPH] from [total NADP]. Similarly, the intracellular levels of GSH, GSSG, and total glutathione (GSH plus GSSG) were measured using the Glutathione Fluorometric Assay Kit (BioVision) according to the manufacturer's specifications.

ROS measurement. For ROS detection, $2 \times 10^{5}$ cells were seeded into 6-well plates; the next day, the medium was replaced with glucose-free medium and maintained for 4-8 hours. Cells were stained with $0.5 \mathrm{mM}$ CM-H2DCF-DA (C6827, Molecular Probes) in PBS for 30 minutes at $37^{\circ} \mathrm{C}$, washed twice with 
PBS, resuspended in $0.4 \mathrm{~mL}$ PBS, and analyzed by flow cytometry to record the green fluorescence of oxidized DCF. The mean fluorescence of 10,000 analyzed cells (compensated with autofluorescence) was taken as readout for the intracellular ROS load.

Statistics. Statistical analysis was performed with SPSS version 20.0 and GraphPad Prism 6. Two-tailed Student's $t$ test was used for single comparisons, and ANOVA with Tukey's multiple-comparisons test was used for multiple comparisons unless otherwise specified. Correlation coefficients were calculated by the PASS Pearson's $\chi^{2}$ test. $P$ values less than 0.05 were considered statistically significant. Data are expressed as mean \pm SEM of $n$ observations.

Study approval. All animal experiments in this study were approved by and adhered to the guidelines of the MD Anderson Cancer Center Animal Care and Use Committee.

\section{Author contributions}

The research was conceived and designed by IKV, MKP, SJS, and MSS.Most experiments were performed by IKV and MKP. SRS and SWC performed human myotube analysis. YY and HK performed immunoprecipitation and in vivo and in vitro ubiquitination assays. $\mathrm{PMB}$ and $\mathrm{VN}$ performed muscle $\mathrm{SDH}$ analysis. CM performed palmitate oxidation analysis. SC and JC performed genetic analysis of $d U B E 2 O$. Data were analyzed by IKV, MKP, SJS, and MSS. The manuscript was written by IKV, MKP, SJS, and MSS.

\section{Acknowledgments}

We thank Mien-Chie Hung, Laura Beretta, Pradip K. Saha, and Pier Paolo Pandolfi for sharing reagents and for critical discussions. We are grateful to Thomas Garvey for critical editing of the manuscript. We thank the MD Anderson Cancer Center Genetically Engineered Mouse Facility and Proteomics Core Facility for technical support and assistance. We thank the Baylor College of Medicine Mouse Metabolism and Phenotyping Core Facility for technical support in mouse metabolism analysis. This work was supported by the National Research Foundation of Korea (NRF) grant funded by the Korean government (MEST) (2018R1A2B6006630) to SJS and an NIH grant (CA196740) to MSS.

Address correspondence to: Su Jung Song, Soonchunhyang Institute of Medi-Bio Science, Soonchunhyang University, 25 Bongjeong-ro, Dongnam-gu, Cheonan-si, Chungcheongnam-do 31151, South Korea. Phone: 82.41.413.5030; Email: ssong1@sch.ac.kr. Or to: Min Sup Song, Department of Molecular and Cellular Oncology, The University of Texas MD Anderson Cancer Center, 1515 Holcombe Blvd., Houston, Texas 77030, USA. Phone: 713.745.4904; Email: msong1@mdanderson.org.

1. Eckel RH, Grundy SM, Zimmet PZ. The metabolic syndrome. Lancet. 2005;365(9468):1415-1428.

2. Utzschneider KM, Kahn SE. Review: the role of insulin resistance in nonalcoholic fatty liver disease. J Clin Endocrinol Metab. 2006;91(12):4753-4761

3. Tseng YH, Cypess AM, Kahn CR. Cellular bioenergetics as a target for obesity therapy. Nat Rev Drug Discov. 2010;9(6):465-482

4. Kahn SE, Hull RL, Utzschneider KM. Mechanisms linking obesity to insulin resistance and type 2 diabetes. Nature. 2006;444(7121):840-846.

5. Saltiel AR, Kahn CR. Insulin signalling and the regulation of glucose and lipid metabolism. Nature. 2001;414(6865):799-806

6. Cline GW, et al. Impaired glucose transport as a cause of decreased insulin-stimulated muscle glycogen synthesis in type 2 diabetes. N Engl J Med. 1999;341(4):240-246.

7. DeFronzo RA, Tripathy D. Skeletal muscle insulin resistance is the primary defect in type 2 diabetes. Diabetes Care. 2009;32(supp1 2):S157-S163.

8. Hardie DG, Ross FA, Hawley SA. AMPK: a nutrient and energy sensor that maintains energy homeostasis. Nat Rev Mol Cell Biol. 2012;13(4):251-262.

9. Viollet B, et al. AMPK: Lessons from transgenic and knockout animals. Front Biosci (Landmark Ed). 2009;14:19-44.

10. Fullerton MD, et al. Single phosphorylation sites in Acc1 and Acc2 regulate lipid homeostasis and the insulin-sensitizing effects of metformin. Nat Med. 2013;19(12):1649-1654.

11. Hunter RW, et al. Metformin reduces liver glucose production by inhibition of fructose-1-6-bisphosphatase. Nat Med. 2018;24(9):1395-1406.

12. Narkar VA, et al. AMPK and PPARdelta agonists are exercise mimetics. Cell. 2008;134(3):405-415.

13. Lee MS, et al. Loss of the E3 ubiquitin ligase MKRN1 represses diet-induced metabolic syndrome through AMPK activation. Nat Commun. 2018;9(1):3404.

14. Pineda CT, et al. Degradation of AMPK by a cancer-specific ubiquitin ligase. Cell. 2015;160(4):715-728.

15. Qi J, et al. Downregulation of AMP-activated protein kinase by Cidea-mediated ubiquitination and degradation in brown adipose tissue. EMBO J. 2008;27(11):1537-1548.

16. Vila IK, et al. A UBE2O-AMPK $\alpha 2$ axis that promotes tumor initiation and progression offers opportunities for therapy. Cancer 
Cell. 2017;31(2):208-224.

17. Hershko A, Ciechanover A. The ubiquitin system. Annu Rev Biochem. 1998;67:425-479.

18. van Wijk SJ, Timmers HT. The family of ubiquitin-conjugating enzymes (E2s): deciding between life and death of proteins. FASEB J. 2010;24(4):981-993.

19. Berleth ES, Pickart CM. Mechanism of ubiquitin conjugating enzyme E2-230K: catalysis involving a thiol relay? Biochemistry. 1996;35(5):1664-1671.

20. Klemperer NS, Berleth ES, Pickart CM. A novel, arsenite-sensitive E2 of the ubiquitin pathway: purification and properties. Biochemistry. 1989;28(14):6035-6041.

21. Mashtalir N, et al. Autodeubiquitination protects the tumor suppressor BAP1 from cytoplasmic sequestration mediated by the atypical ubiquitin ligase UBE2O. Mol Cell. 2014;54(3):392-406.

22. Hao YH, et al. Regulation of WASH-dependent actin polymerization and protein trafficking by ubiquitination. Cell. 2013;152(5):1051-1064.

23. Liang K, et al. Therapeutic targeting of MLL degradation pathways in MLL-rearranged leukemia. Cell. 2017;168(1-2):59-72.e13.

24. Nguyen AT, et al. UBE2O remodels the proteome during terminal erythroid differentiation. Science. 2017;357(6350):eaan0218

25. Yanagitani K, Juszkiewicz S, Hegde RS. UBE2O is a quality control factor for orphans of multiprotein complexes. Science. 2017;357(6350):472-475.

26. Zhang X, et al. Fine-tuning BMP7 signalling in adipogenesis by UBE2O/E2-230K-mediated monoubiquitination of SMAD6. EMBO J. 2013;32(7):996-1007.

27. Zhang X, Zhang J, Zhang L, van Dam H, ten Dijke P. UBE2O negatively regulates TRAF6-mediated NF- $\kappa$ B activation by inhibiting TRAF6 polyubiquitination. Cell Res. 2013;23(3):366-377.

28. Yokota T, et al. Identification, tissue expression, and chromosomal position of a novel gene encoding human ubiquitin-conjugating enzyme E2-230k. Gene. 2001;267(1):95-100.

29. Klop B, Elte JW, Cabezas MC. Dyslipidemia in obesity: mechanisms and potential targets. Nutrients. 2013;5(4):1218-1240.

30. Fabbrini E, Sullivan S, Klein S. Obesity and nonalcoholic fatty liver disease: biochemical, metabolic, and clinical implications Hepatology. 2010;51(2):679-689.

31. Zurlo F, Larson K, Bogardus C, Ravussin E. Skeletal muscle metabolism is a major determinant of resting energy expenditure. J Clin Invest. 1990;86(5):1423-1427.

32. Eguchi J, et al. Transcriptional control of adipose lipid handling by IRF4. Cell Metab. 2011;13(3):249-259.

33. Miniou P, Tiziano D, Frugier T, Roblot N, Le Meur M, Melki J. Gene targeting restricted to mouse striated muscle lineage. Nucleic Acids Res. 1999;27(19):e27.

34. Postic C, et al. Dual roles for glucokinase in glucose homeostasis as determined by liver and pancreatic beta cell-specific gene knock-outs using Cre recombinase. J Biol Chem. 1999;274(1):305-315.

35. Wu Z, et al. Mechanisms controlling mitochondrial biogenesis and respiration through the thermogenic coactivator PGC-1. Cell 1999;98(1):115-124

36. Jeon SM, Chandel NS, Hay N. AMPK regulates NADPH homeostasis to promote tumour cell survival during energy stress. Nature. 2012;485(7400):661-665.

37. Nakada D, Saunders TL, Morrison SJ. Lkb1 regulates cell cycle and energy metabolism in haematopoietic stem cells. Nature 2010;468(7324):653-658.

38. Briffa R, et al. Multi-scale genomic, transcriptomic and proteomic analysis of colorectal cancer cell lines to identify novel biomarkers. PLoS ONE. 2015;10(12):e0144708.

39. Lin M, et al. DNA copy number gains in head and neck squamous cell carcinoma. Oncogene. 2006;25(9):1424-1433.

40. Rice KL, et al. Analysis of genomic aberrations and gene expression profiling identifies novel lesions and pathways in myeloproliferative neoplasms. Blood Cancer J. 2011;1(11):e40.

41. Toffoli S, et al. Identification by array comparative genomic hybridization of a new amplicon on chromosome $17 \mathrm{q}$ highly recurrent in BRCA1 mutated triple negative breast cancer. Breast Cancer Res. 2014;16(6):466.

42. Wang $X$, et al. Copy number alterations detected by whole-exome and whole-genome sequencing of esophageal adenocarcinoma. Hum Genomics. 2015;9:22.

43. Liesa M, Shirihai OS. Mitochondrial dynamics in the regulation of nutrient utilization and energy expenditure. Cell Metab. 2013;17(4):491-506.

44. Cypess AM, et al. Activation of human brown adipose tissue by a $\beta 3$-adrenergic receptor agonist. Cell Metab. 2015;21(1):33-38.

45. Mullur R, Liu YY, Brent GA. Thyroid hormone regulation of metabolism. Physiol Rev. 2014;94(2):355-382.

46. Mu J, Brozinick JT Jr. Valladares O, Bucan M, Birnbaum MJ. A role for AMP-activated protein kinase in contraction- and hypoxia-regulated glucose transport in skeletal muscle. Mol Cell. 2001;7(5):1085-1094.

47. Vichaiwong K, et al. Contraction regulates site-specific phosphorylation of TBC1D1 in skeletal muscle. Biochem J. 2010;431(2):311-320.

48. Minokoshi Y, et al. Leptin stimulates fatty-acid oxidation by activating AMP-activated protein kinase. Nature. 2002;415(6869):339-343.

49. Yamauchi T, et al. Adiponectin stimulates glucose utilization and fatty-acid oxidation by activating AMP-activated protein kinase. Nat Med. 2002;8(11):1288-1295.

50. Jørgensen SB, et al. Effects of alpha-AMPK knockout on exercise-induced gene activation in mouse skeletal muscle. FASEB J. 2005;19(9):1146-1148

51. Bentzinger CF, et al. Differential response of skeletal muscles to mTORC1 signaling during atrophy and hypertrophy. Skelet Muscle. 2013;3(1):6.

52. Bentzinger CF, et al. Skeletal muscle-specific ablation of raptor, but not of rictor, causes metabolic changes and results in muscle dystrophy. Cell Metab. 2008;8(5):411-424

53. Masiero E, et al. Autophagy is required to maintain muscle mass. Cell Metab. 2009;10(6):507-515.

54. Castets $\mathrm{P}$, et al. Sustained activation of $\mathrm{mTORC} 1$ in skeletal muscle inhibits constitutive and starvation-induced autophagy and causes a severe, late-onset myopathy. Cell Metab. 2013;17(5):731-744. 
55. Um SH, et al. Absence of S6K1 protects against age- and diet-induced obesity while enhancing insulin sensitivity. Nature. 2004;431(7005):200-205.

56. Harrington LS, et al. The TSC1-2 tumor suppressor controls insulin-PI3K signaling via regulation of IRS proteins. J Cell Biol. 2004; 166(2):213-223

57. Barré L, et al. Genetic model for the chronic activation of skeletal muscle AMP-activated protein kinase leads to glycogen accumulation. Am J Physiol Endocrinol Metab. 2007;292(3):E802-E811.

58. Barnes BR, et al. The 5'-AMP-activated protein kinase gamma3 isoform has a key role in carbohydrate and lipid metabolism in glycolytic skeletal muscle. J Biol Chem. 2004;279(37):38441-38447.

59. Yu H, Hirshman MF, Fujii N, Pomerleau JM, Peter LE, Goodyear LJ. Muscle-specific overexpression of wild type and R225Q mutant AMP-activated protein kinase gamma3-subunit differentially regulates glycogen accumulation. Am J Physiol Endocrinol Metab. 2006;291(3):E557-E565.

60. Salt I, et al. AMP-activated protein kinase: greater AMP dependence, and preferential nuclear localization, of complexes containing the alpha2 isoform. Biochem J. 1998;334(pt 1):177-187.

61. Stapleton D, et al. Mammalian AMP-activated protein kinase subfamily. J Biol Chem. 1996;271(2):611-614.

62. Wojtaszewski JF, Birk JB, Frosig C, Holten M, Pilegaard H, Dela F. 5'AMP activated protein kinase expression in human skeletal muscle: effects of strength training and type 2 diabetes. J Physiol. 2005;564(pt 2):563-573.

63. Woods A, Salt I, Scott J, Hardie DG, Carling D. The alpha1 and alpha2 isoforms of the AMP-activated protein kinase have similar activities in rat liver but exhibit differences in substrate specificity in vitro. FEBS Lett. 1996;397(2-3):347-351.

64. Friedrichsen M, Mortensen B, Pehmøller C, Birk JB, Wojtaszewski JF. Exercise-induced AMPK activity in skeletal muscle: role in glucose uptake and insulin sensitivity. Mol Cell Endocrinol. 2013;366(2):204-214.

65. Richter EA, Ruderman NB. AMPK and the biochemistry of exercise: implications for human health and disease. Biochem J. 2009;418(2):261-275.

66. Cokorinos EC, et al. Activation of skeletal muscle AMPK promotes glucose disposal and glucose lowering in non-human primates and mice. Cell Metab. 2017;25(5):1147-1159.e10.

67. Myers RW, et al. Systemic pan-AMPK activator MK-8722 improves glucose homeostasis but induces cardiac hypertrophy. Science. 2017;357(6350):507-511.

68. Chen $\mathrm{H}$, et al. Evidence that the diabetes gene encodes the leptin receptor: identification of a mutation in the leptin receptor gene in $\mathrm{db} / \mathrm{db}$ mice. Cell. 1996;84(3):491-495.

69. Vila IK, et al. Immune cell Toll-like receptor 4 mediates the development of obesity- and endotoxemia-associated adipose tissue fibrosis. Cell Rep. 2014;7(4):1116-1129.

70. Narkar VA, et al. Exercise and PGC-1 $\alpha$-independent synchronization of type I muscle metabolism and vasculature by ERR $\gamma$. Cell Metab. 2011;13(3):283-293. 